\title{
TETs compete with DNMT3 activity in pluripotent cells at thousands of methylated somatic
} enhancers

\author{
Jocelyn Charlton ${ }^{1,2,3}$, Eunmi J. Jung1, Alexandra L. Mattei ${ }^{1,3,4}$, Nina Bailly ${ }^{3,5}$, Jing Liao', \\ Eric J. Martin ${ }^{6,7}$, Pay Giesselmann $\mathbb{1 0}^{3}$, Björn Brändl ${ }^{3}$, Elena K. Stamenova², Franz-Josef Müller ${ }^{3,8}$, \\ Evangelos Kiskinis ${ }^{6,7}$, Andreas Gnirke ${ }^{2}$, Zachary D. Smith ${ }^{1,2}$ and Alexander Meissner ${ }^{1,2,3,5 \bowtie}$
}

\begin{abstract}
Mammalian cells stably maintain high levels of DNA methylation despite expressing both positive (DNMT3A/B) and negative (TET1-3) regulators. Here, we analyzed the independent and combined effects of these regulators on the DNA methylation landscape using a panel of knockout human embryonic stem cell (ESC) lines. The greatest impact on global methylation levels was observed in DNMT3-deficient cells, including reproducible focal demethylation at thousands of normally methylated loci. Demethylation depends on TET expression and occurs only when both DNMT3s are absent. Dynamic loci are enriched for hydroxymethylcytosine and overlap with subsets of putative somatic enhancers that are methylated in ESCs and can be activated upon differentiation. We observe similar dynamics in mouse ESCs that were less frequent in epiblast stem cells (EpiSCs) and scarce in somatic tissues, suggesting a conserved pluripotency-linked mechanism. Taken together, our data reveal tightly regulated competition between DNMT3s and TETs at thousands of somatic regulatory sequences within pluripotent cells.
\end{abstract}

M ammalian genomes generally display high levels of $\mathrm{CpG}$ methylation, with the exception of most CpG-dense promoter regions, as well as certain enhancers that are either developmentally poised, actively engaged or decomissioned ${ }^{1}$. Somatic DNA methylation landscapes are overall stably propagated, with alterations to these global patterns linked to aging and disease $^{2-5}$. In contrast, developmental regulation of $\mathrm{CpG}$ methylation during cellular differentiation generally reflects focal reprogramming in response to transcription factor binding ${ }^{6}$.

The de novo DNA methyltransferases DNMT3A and DNMT3B are responsible for addition of a methyl group to generate 5 -methylcytosine, while DNMT1 is largely dedicated to propagating pre-established modifications over DNA replication. In contrast, the Ten-eleven translocation (TET1, TET2 and TET3) enzymes oxidize 5 -methylcytosine to generate 5 -hydroxymethylcytosine $(5 \mathrm{hmC})$ or further oxidized species ${ }^{7-9}$ that can then be either passively or enzymatically removed from $\mathrm{DNA}^{10,11}$.

Both de novo methylation and demethylation are essential for normal development ${ }^{12}$, and single knockouts result in embryonic (DNMT1, DNMT3B) or early postnatal (DNMT3A, TET3) lethality $^{13-15}$. Single and combined knockouts of TET1 and TET2 are largely viable but present a wide range of abnormalities ${ }^{16}$. Loss-of-function studies have been instrumental in assigning specific roles for each enzyme. For instance, DNMT3B is preferentially recruited to satellite repeats and transcriptionally active gene bodies ${ }^{17,18}$. DNMT3A appears to function at the periphery of hypomethylated enhancers, over extended hypomethylated domains termed 'canyons' and at developmentally poised promoters ${ }^{19,20}$, where TET1 and TET2 act in opposition to maintain a hypomethylated state ${ }^{20-25}$. TET2 may also have additional functions to regulate transcriptional elongation across gene bodies ${ }^{26}$.

DNMT1, DNMT3A and DNMT3B are expressed in human ESCs, along with TET1, TET2 and TET3, which differs from mouse ESCs, where TET3 is typically not expressed. Human ESCs require the expression of catalytically active DNMT1 to remain viable, while knockout of DNMT3A and -3B gives rise to morphologically normal cells that slowly lose methylation over time ${ }^{18}$. Conversely, human ESCs that lack all three TET enzymes appear to maintain global methylation levels, but show focal gains and losses of methylation, including hypermethylation of developmental gene promot$\mathrm{ers}^{21}$. While both DNMT3- and TET-free scenarios have been well described, to the best of our knowledge no study has yet directly compared the impact of losing all demethylating and methylating capabilities on transcriptional and genome regulation as well as cellular viability.

To examine the molecular consequences of removing all active regulators of DNA methylation, we generated pentuple-knockout (PKO) human ESCs deficient in both DNMT3A and -3B, as well as all three TETs. Altogether, we compared genome-wide methylation levels across fourteen different wild-type (WT) and knockout human ESC lines. Importantly, we find that TET enzymes are focally recruited to thousands of somatic enhancers throughout the genome that nonetheless remain highly methylated in pluripotent cells as long as either DNMT3 is present. TETs also have a more widespread activity throughout the genome that requires DNMT3 expression to maintain steady-state methylation levels. Together,

'Department of Stem Cell and Regenerative Biology, Harvard University, Cambridge, MA, USA. 'Broad Institute of MIT and Harvard, Cambridge, MA, USA. ${ }^{3}$ Department of Genome Regulation, Max Planck Institute for Molecular Genetics, Berlin, Germany. ${ }^{4}$ Department of Molecular and Cellular Biology, Harvard University, Cambridge, MA, USA. Institute of Chemistry and Biochemistry, Freie Universität Berlin, Berlin, Germany. ${ }^{6}$ The Ken \& Ruth Davee Department of Neurology, Northwestern University Feinberg School of Medicine, Chicago, IL, USA. ${ }^{7}$ Department of Physiology, Northwestern University

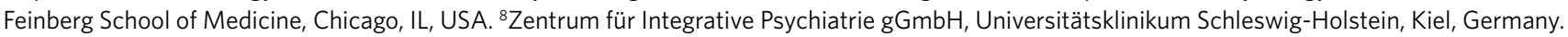
凶e-mail: meissner@molgen.mpg.de 
our results suggest both focal and global competition for each enzyme class.

\section{Results}

TETs drive global demethylation in the absence of DNMT3s. To complement earlier studies that investigated DNMT or TET loss in human ESCs, we set out to derive the full range of knockouts and comprehensively assess the interplay between methylating and demethylating enzymes. To this end, we took WT HUES8 ESCs and used Cas9 with single-guide RNAs (sgRNAs) targeted to DNMT3A and DNMT3B to derive HUES8 double-knockout (DKO) ESCs that lack all de novo methyltransferase activity. We also obtained previously generated HUES8 ESCs that were depleted of all TET activity, $T E T 1^{-1-}, T E T 2^{-/-}, T E T 3^{-1-}$ triple-knockout (TKO) ESCs, as well as quadruple-knockout (QKO) ESCs which are derived from the TKO with additional knockout of DNMT3B (ref. ${ }^{21}$ ). We then established a new cell line by further mutating DNMT3A within the HUES8 QKO to generate DNMT3 $A^{-1}, D N M T 3 B^{-1-}, T E T 1^{-1-}$, TET2 ${ }^{-/-}$, TET3 $^{-/-}$PKO ESCs (Fig. 1a, top, and Extended Data Fig. 1a-c). All knockout cell lines appeared morphologically normal and expressed self-renewal- and pluripotency-associated genes, suggesting that DNMT3 and TET expression is not required within the undifferentiated state (Extended Data Fig. 1d-f). We then performed whole-genome bisulfite sequencing (WGBS) to investigate global DNA methylation patterns within these lines, recovering 6.7 million matched CpGs with $\geq 10 \times$ coverage across all samples. As expected, HUES8 WT showed high mean CpG methylation (WT, 0.78; Fig. 1a, bottom). Global methylation levels decreased by 0.09 within our DNMT3-null ESCs after six passages (DKO, 0.69) compared with a global mean increase of only 0.01 in the TET-null ESCs (TKO, 0.79). Interestingly, when the de novo methyltransferases were knocked out in TET-null cells, we observed little to no change at passage $6(\mathrm{QKO}, 0.78$; $\mathrm{PKO}, 0.77)$. These results suggest that the majority of DNA demethylation following DNMT3 depletion depends on TET activity.

Focal competition between DNMTs and TETs in pluripotent cells. To investigate this methylation loss further, we next sequenced WT and DKO cells from an additional human ESC line (HUES64), including two independent $\mathrm{DKO}$ clones (A and $\mathrm{B}$ ), and compared methylation levels with previously derived ${ }^{18}$ single-DNMT3 $A^{-1-}$ $(3 \mathrm{AKO})$ and single-DNMT3B ${ }^{-/-}$(3BKO) knockout data (Fig. 1b). The two different male WT ESCs appear comparable, with similar global means (HUES64, 0.79; HUES8, 0.78) and high correlation over 1-kilobase $(\mathrm{kb})$ tiles (Pearson coefficient $=0.93$; Extended Data Fig. 1g). Interestingly, while single-DNMT3 knockouts at passage 22 remained highly methylated $(3 \mathrm{AKO}, 0.81 ; 3 \mathrm{BKO}, 0.79)$, we again observed a methylation decrease to 0.70 after only three passages for both HUES64 DKO clones (Fig. 1b). When we examined our data at base-pair resolution, we observed two distinct dynamic categories: a subtle decrease of $0.1-0.2$ that affected $\sim 10 \%$ of CpGs genome-wide, as well as nearly complete loss of methylation that occurs locally within CpG clusters (Fig. 1c and Extended Data Fig. 2a). We were particularly intrigued by these extreme focal changes and proceeded to define differentially methylated regions (DMRs) using stringent criteria (methylation difference $>0.6, P<0.01, F$-test). DKO-DMRs were remarkably consistent across our three DKO lines (Fig. 1c,d; $P<10^{-16}$, hypergeometric test). We therefore defined a 'consensus' set of 11,430 cDKO-DMRs that were hypomethylated in all three samples (Supplementary Table 1). cDKO-DMRs maintain consistently low levels (mean WT, 0.749; DKO,0.086), and average 688 base pairs (bp) in length. Approximately one-third neighbor already hypomethylated regions in WT ESCs, such as CpG islands and transcription start sites (termed 'class 1'), while the rest are located in otherwise highly methylated regions (intronic or intergenic) with distinct hypermethylated borders (termed 'class 2';
Fig. 1e-h). Almost all cDKO-DMRs (93\%) were redundantly methylated by either DNMT3A or -3B, and only became fully demethylated in the DKO (Fig. $1 \mathrm{i}$ and Extended Data Fig. 2b). As these results strongly suggest an active demethylation mechanism, we studied methylation levels for cDKO-DMRs across the TET-depleted HUES8 cell lines (TKO, QKO and PKO). In the absence of TETs, the subsequent knockout of first DNMT3B and then DNMT3A did not result in cDKO-DMR demethylation (Fig. 1i). Even after 20 passages, cDKO-DMR methylation levels decreased only minimally in PKO cells (Extended Data Fig. 2c). Moreover, the areas surrounding many class $1 \mathrm{cDKO}-\mathrm{DMRs}$ gained methylation in TKO cells, further implicating TET activity in the focal demethylation (Extended Data Fig. 2d). Together, our data highlight a set of distinct and highly methylated regions in human pluripotent cells that rely on DNMT3A or -3B and undergo rapid TET-mediated demethylation in their absence.

TETs display continuous activity at cDKO-DMRs. To verify that cDKO-DMRs depend on and continue to attract TET activity, we utilized PiggyBac transposition to reintroduce exogenous TET1 (the short isoform, hereafter referred to as TET1s), TET2 and TET3 into PKO cells and measured global methylation levels using WGBS (Fig. 2a). We observed a clear reduction in global mean methylation from 0.73 in PKO cells at passage 20 to $0.61,0.59$ and 0.54 after ectopically expressing each protein, while control cells transfected with only the transposase enzyme remained stable (mean $=0.70$; Fig. 2b). Reintroducing any TET enzyme largely restored aberrantly hypermethylated regions in TKO cells and induced rapid cDKO-DMR demethylation, demonstrating that their recruitment is highly redundant and preserved even after extended periods without TET activity (Fig. 2c-e and Extended Data Fig. 2e). From a mechanistic standpoint, it is worth noting that the TET1s isoform lacks a CXXC domain for recruitment to unmethylated CpG -dense regions ${ }^{27-29}$, yet we found no obvious difference between the TET1s and TET2 rescue, supporting a CXXC domain-independent recruitment mechanism (Extended Data Fig. 2f). Ongoing dynamics between TETs and their opposing enzymes at CDKO-DMRs is also supported by published chromatin immunoprecipitation followed by sequencing (ChIP-seq) data, which show enrichment for both DNMT3B and TET1 in WT ESCs, although enrichment appears broader than the rather narrow and well-defined cDKO-DMR boundaries (Extended Data Fig. 2g). Finally, we performed whole-genome oxidative bisulfite sequencing (oxBS) and found significant local enrichment of $5 \mathrm{hmC}$ in WT cells (mean cDKO-DMRs, 0.07; background, 0.01; $P=2.2 \times 10^{-16}$, two-sided $t$-test, $t=231.8$, d.f. $=255,010$; Fig. 2 f and Extended Data Fig. 2h). Put together, these results confirm that TET enzymes are continuously recruited to thousands of loci throughout the genome and can direct rapid, focal demethylation if DNMT3 activity is absent.

Dynamic DNMT-TET recruitment at evolutionarily young retrotransposons. The robust and precise manner by which our cDKO-DMRs change their methylation state suggests that they may overlap with regulatory elements. Notably, a small subset includes the $5^{\prime}$ untranslated region (UTR) of L1Hs or L1PA long interspersed nuclear elements (LINEs), which act as their functional promoter and generally display evolutionary age- and $\mathrm{CpG}$ density-dependent demethylation following DNMT3 knockout (Fig. 3a,b and Extended Data Fig. 3a,b). These regions also show higher than expected $5 \mathrm{hmC}$ enrichment in WT ESCs, supporting continual TET recruitment (Fig. 3b). To investigate this further, we focused on the human-specific L1Hs elements and used the MinION platform from Oxford Nanopore to generate long reads (mean length $=8.9 \mathrm{~kb}$ ) that enable unambiguous mapping and assessment of LINE-specific methylation states for HUES64 WT and DKO ESCs, as well as HUES8 WT, TKO and PKO ESCs (Extended 


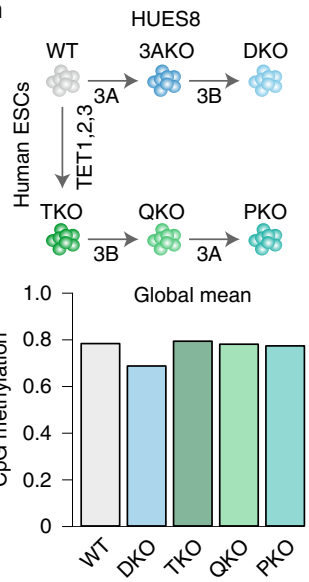

b

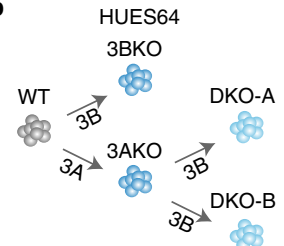

C $\quad \operatorname{chr} 12: 124,900,840-124,946,350$

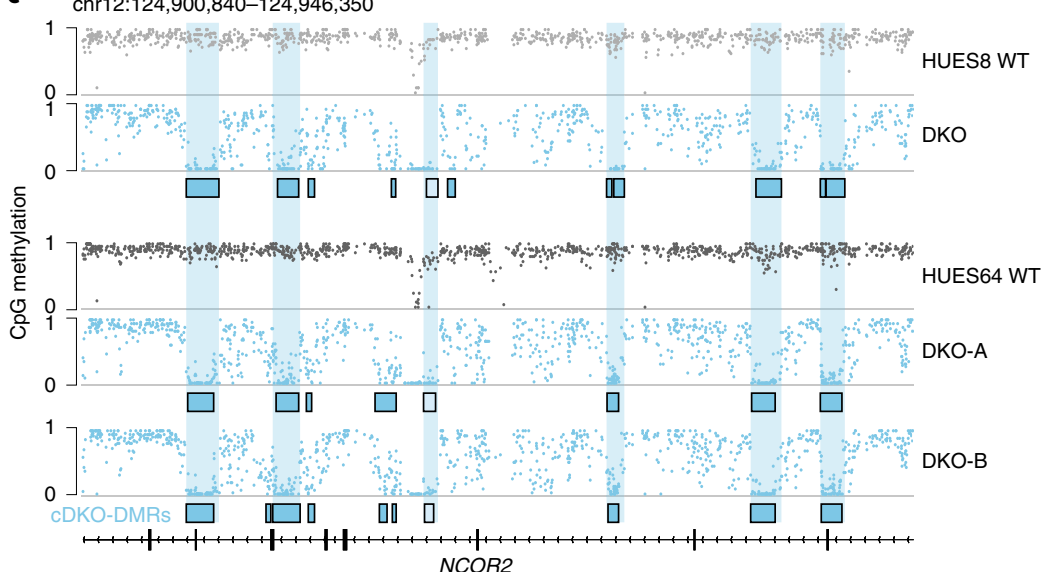

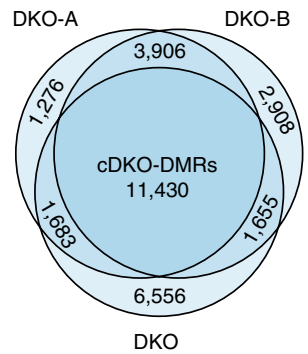
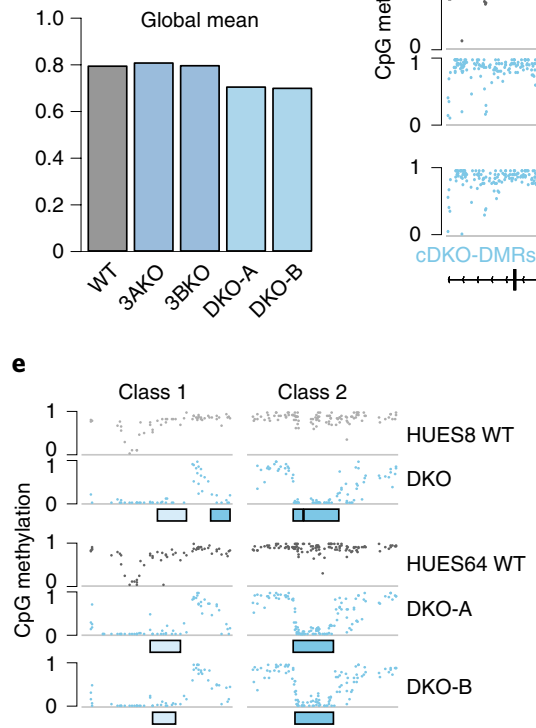

f

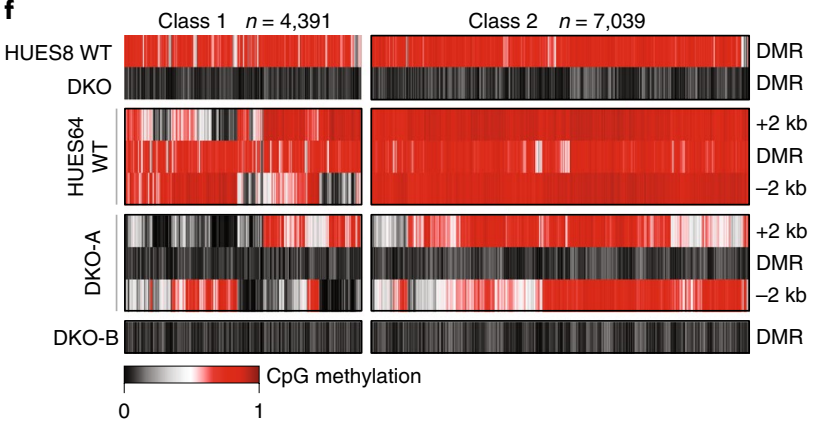

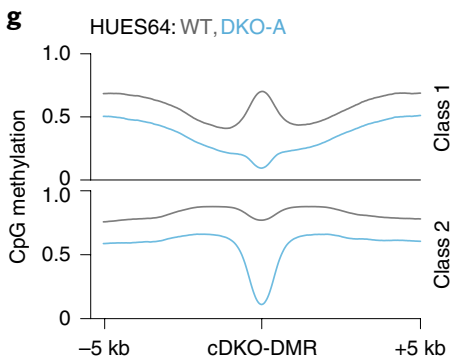
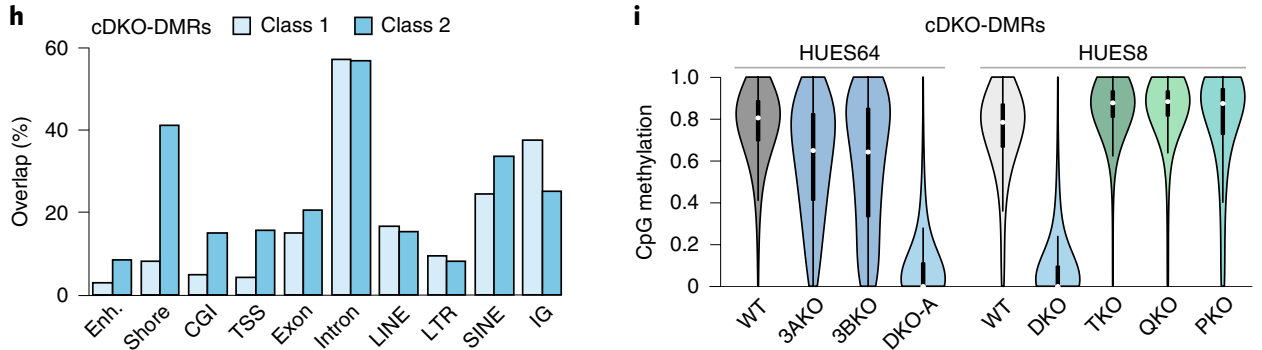

Fig. 1 | Focal demethylation by TET enzymes in the absence of DNMT3s. a, Schematic overview. Knockouts are shown under each arrow. 3A, DNMT3A; 3B, DNMT3B. Bar plot shows the global mean methylation for each sample using matched CpGs $(n=6,708,067)$ generated by WGBS. DKO and PKO ESCs were passaged six times. b, Schematic as in a. Bar plot shows the global mean methylation for each sample (CpGs match panel a). DKO-A and DKO-B denote clone A and B (passage 3), respectively. $3 A$ KO and 3BKO are from published datasets ${ }^{18}$. c, Genome browser tracks displaying WT and DKO methylation levels, sample-specific DKO-DMRs (boxes) and shared cDKO-DMRs (shaded vertical bars). d, The overlap between DKO-DMRs hypomethylated in each sample. e, Genome browser track of selected DKO-DMRs shown in panel c. Class 1 (light shading) border already hypomethylated regions and class 2 (dark shading) are flanked by highly methylated DNA. f, Heatmap of cDKO-DMRs including $2 \mathrm{~kb}$ on either side, separated by class. $\mathbf{g}$, Methylation composite plot of class 1 and class 2 cDKO-DMRs with $5 \mathrm{~kb}$ on either side. $\mathbf{h}$, The percentage of cDKO-DMRs that overlap with the shown genomic features. Categories are not exclusive. CGI, CpG island; TSS, transcription start site; IG, intergenic. Enhancers are defined from H1 ESCs ${ }^{32}$. i, Violin plots for CpGs located within cDKO-DMRs ( $n=214,732,180,019,138,424,215,263,203,504,182,322,202,028,212,215,145,869$ CpGs, respectively). Plots extend from the data minima to the maxima with the white dot indicating median, the thick bar showing the interquartile range and the thin bar showing $1.5 \times$ interquartile range.

Data Fig. 3c). In WT cells, we found that $7 \%$ of L1Hs $5^{\prime}$ UTRs ( $n=207$ full length covered in each sample) were already hypomethylated (Fig. 3c). Despite often not passing our stringent DMR criteria, $91 \%$ of WT methylated elements were demethylated following DNMT3A and DNMT3B knockout (Fig. 3c). Demethylation was dependent on TET expression, with only a small group showing loss in PKO cells (Fig. 3c). Notably, we could not pinpoint any single-nucleotide polymorphism that was consistent with methylation state after partitioning single LINEs according to their methylation dynamics, suggesting a more complex mechanism behind DNMT/TET recruitment (Extended Data Fig. 3d,e). Of note, the
17 L1Hs 5' UTRs that remained methylated in DKO ESCs have a lower CpG density and GC content, which may indicate that higher CpG density favors TET recruitment and demethylation (Extended Data Fig. 3f). Cumulatively, it appears that LINE 5' UTRs are highly sensitive to DNMT3/TET expression and can switch methylation states rapidly, yet the methylated state is favored for most elements in DNMT3-expressing pluripotent cells.

Somatic enhancers are targeted by DNMT3 and TET activity in pluripotent cells. While TETs can be recruited to LINE $5^{\prime} \mathrm{UTRs}^{30}$, they more frequently localize to promoters or active enhancers 

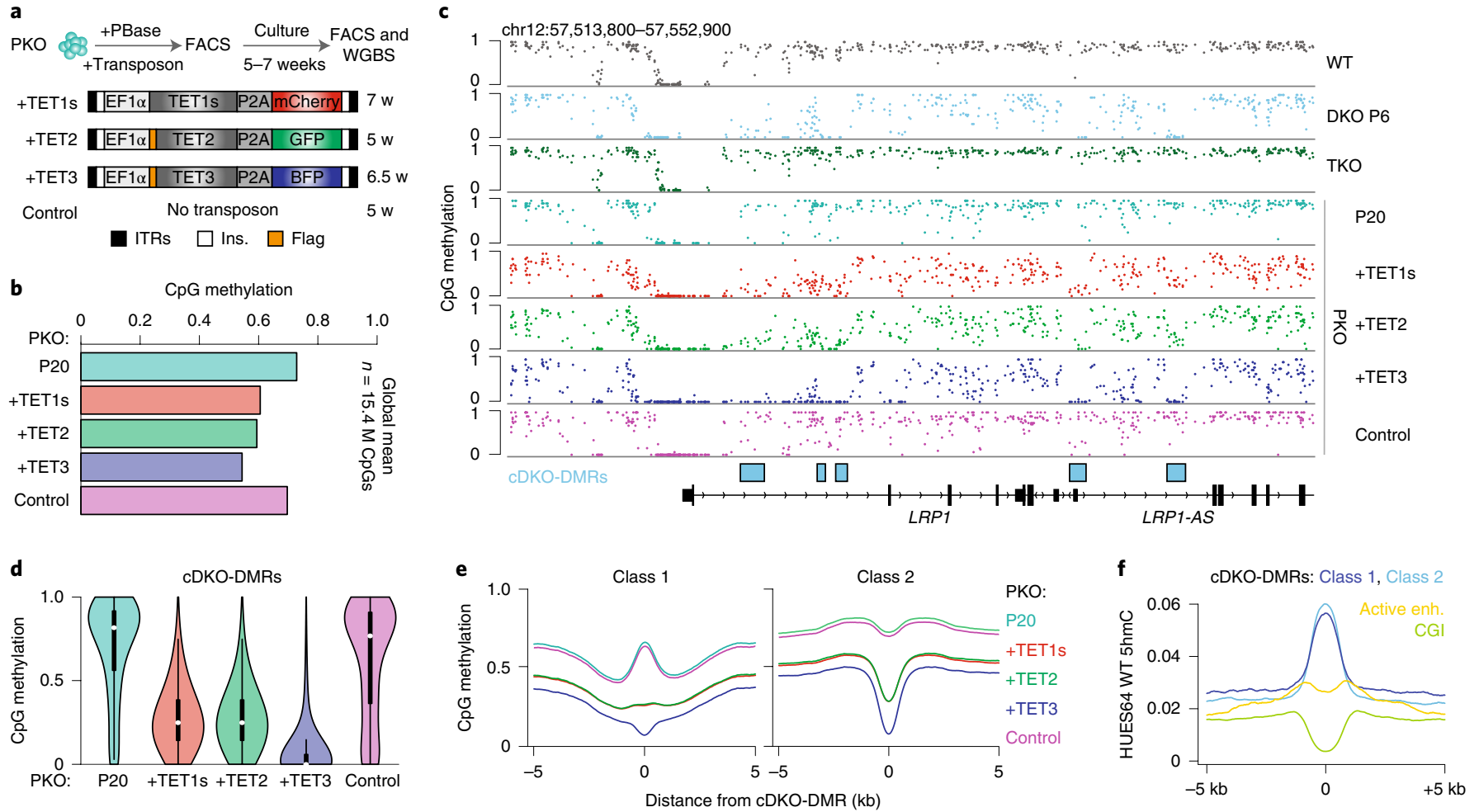

Fig. 2 | Re-expression of TET enzymes demethylates cDKO-DMRs in PKO cells. a, TET1s, TET2 and TET3 were reintroduced into PKO cells using PiggyBac transposition. Control transfection was performed with only the transposase (PBase). A schematic diagram depicts the experimental design with the constructs shown below; w, weeks; Ins, insulator sequence. b, WGBS-based mean methylation bar plots for matched CpGs. P20, passage 20. c, Genome browser tracks across a 39-kb region of the LRP1 locus for HUES8 WT and seven of the modified HUES8 lines. Re-expression of TET1s, TET2 or TET3 in PKO cells causes global and specific loss of methylation at cDKO-DMRs (blue boxes). d, Violin plots displaying methylation levels for CpGs within CDKO-DMRs ( $n=176,669,186,438,155,912,180,233,128,709$ CpGs, respectively). Violin plots extend from the data minima to the data maxima with the white dot indicating median, thick bar showing the interquartile range and thin bar showing $1.5 \times$ interquartile range. e, Composite plot of mean methylation levels for each class of cDKO-DMR, including $5 \mathrm{~kb}$ on either side for PKO cells at P2O and control cells, as well as the three TET rescue lines. f, Mean hydroxymethylcytosine $(5 \mathrm{hmC})$ levels in WT HUES64 cells shown as distance from class 1 or class 2 cDKO-DMR center, CGIs or active ESC $(\mathrm{H} 1)$ enhancers (enh).

(Extended Data Fig. 4a), where they maintain the hypomethylated state $\mathrm{e}^{23}$. Indeed $7 \%$ of cDKO-DMRs neighbor hypomethylated active enhancers, supporting previous evidence that DNMT3s protect enhancer boundaries from extended hypomethylation ${ }^{20,31}$ (Fig. 3a,d,e). Given a canonical role for TETs at active enhancers, we reasoned that $\mathrm{CDKO}-\mathrm{DMRs}$ may also represent regulatory elements that only undergo demethylation when they are activated in alternative cell states. Strikingly, $85 \%$ of cDKO-DMRs overlap with at least one previously defined tissue-specific putative enhancer ${ }^{32}$ and are specifically demethylated in the associated cell type (Fig. 3a,f,g and Extended Data Fig. 4b,c; controlled background overlap 35\%). Interestingly, while cDKO-DMRs were almost always somatic enhancers, the inverse was not true: many somatic enhancers are already hypomethylated in ESCs, remain highly methylated in their associated tissue or do not display TET-dependent demethylation in ESCs despite losing methylation in other developmental contexts (Fig. 3h and Extended Data Fig. 4d). To explore DMR stability, we further passaged HUES64 DKO clone A cells and performed WGBS. After 28 passages without DNMT3 activity, we identified an increased number of 59,618 DKO-DMRs, $79 \%$ of which overlapped with putative tissue-specific enhancers (Extended Data Fig. 4e). Nonetheless, approximately one-third of somatic enhancers remained methylated in late-passage DKO cells (Extended Data Fig. 4f). To examine whether the ability to demethylate an enhancer is linked to developmental timing (when they become activated during development), we utilized a recently published dataset detailing enhancer activation and methylation dynamics over nine stages of pancreatic islet differentiation from HUES64 $\mathrm{ESCs}^{33}$ (Extended Data Fig. 4g). Interestingly, a similar proportion of enhancers at every stage of differentiation overlapped with DKO-DMRs, with a slightly higher frequency at more-terminal differentiation stages (Extended Data Fig. 4g-i). Although speculative, the lack of an obvious connection to ESC differentiation suggests that a subset of both embryonic and adult tissue-specific enhancers may exist in a state that depends on the continuous and opposing functions of DNMT3s and TETs.

Loss of DNA methylation at somatic enhancers in ESCs affects gene expression. We next wondered how TETs are recruited to this set of highly methylated somatic enhancers and what the impact of losing active DNMT3 recruitment could be. cDKO-DMRs have a greater than background CpG density (3.2\%), yet this is not classifying since the majority of $1-\mathrm{kb}$ tiles with matched $\mathrm{CpG}$ density do not lose methylation in DKO cells (Extended Data Fig. 5a,b). When we looked for associations between CDKO-DMRs and selected epigenetic features in WT ESCs, we found that they were generally more enriched for open chromatin and H3K4me1, but not H3K27ac, which is commonly associated with transcriptionally engaged 'active' enhancers (Fig. 3i and Extended Data Fig. 5c-e). Although frequently overlapping, $\mathrm{H} 3 \mathrm{~K} 4 \mathrm{mel}$ enrichment was again not sufficient to predict cDKO-DMRs (Extended Data Fig. 5f). Further, only $\sim 10 \%$ of $\mathrm{H} 3 \mathrm{~K} 4 \mathrm{mel}$-enriched somatic enhancers were also 

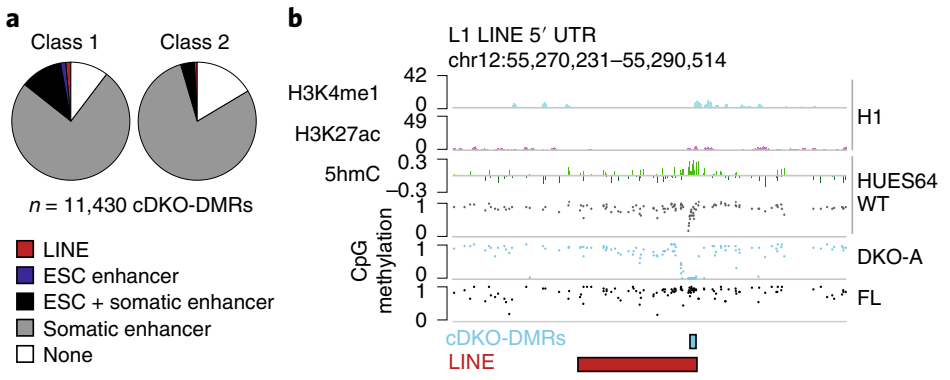
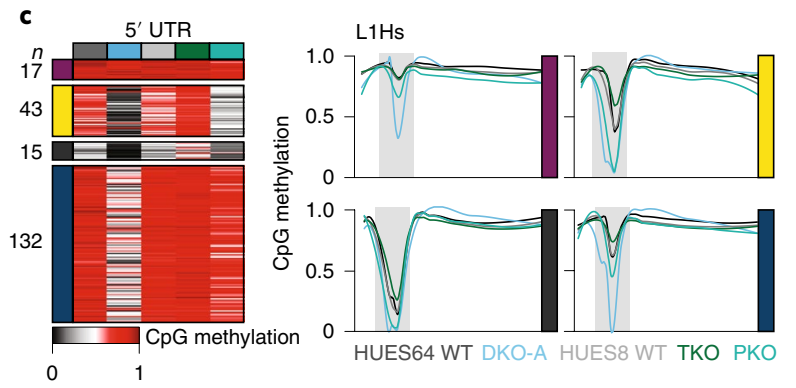
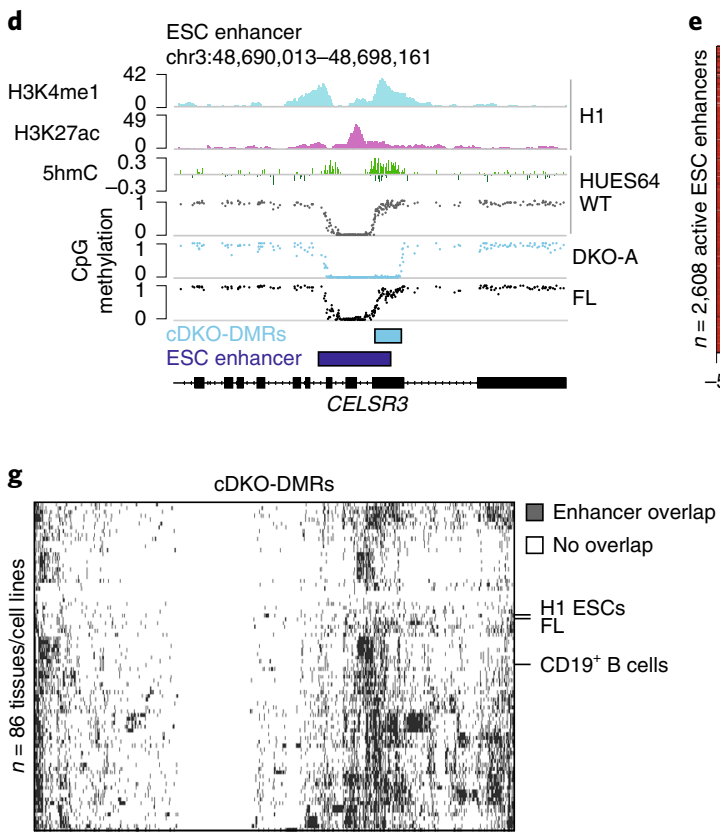
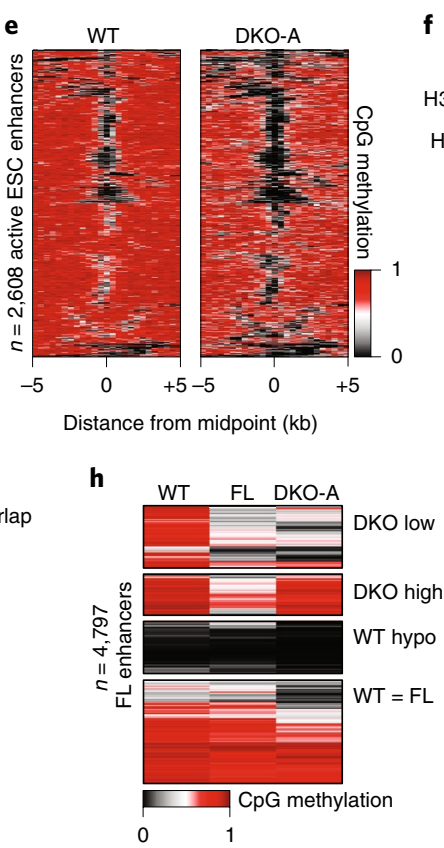

i
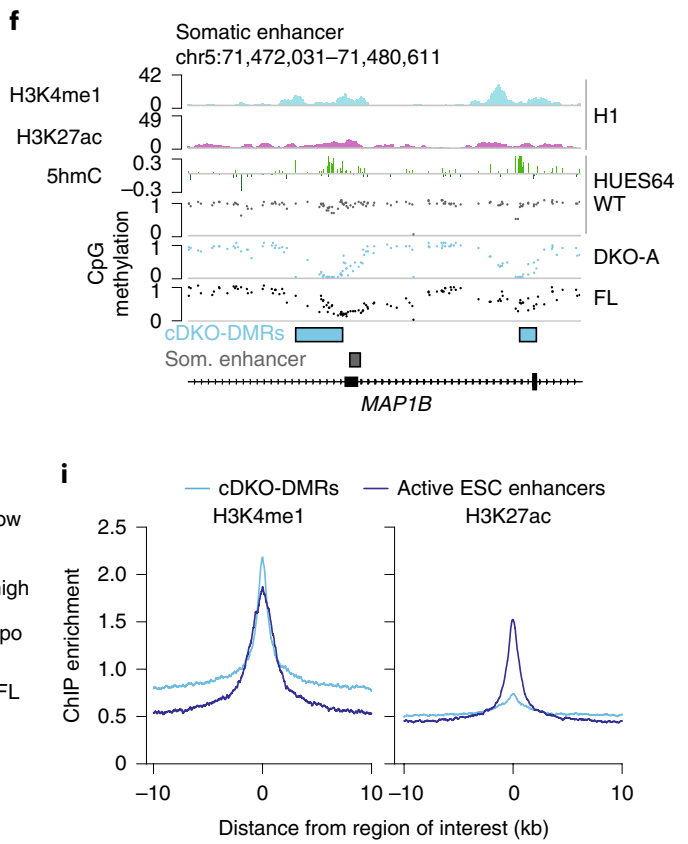

Fig. 3 | TETs demethylate LINEs and somatic enhancers following DNMT3 loss. a, The proportion of class 1 or class 2 cDKO-DMRs that overlap with selected features. b, Genome browser tracks showing cDKO-DMR overlap within the 5' UTR of a human LINE (L1PA3) element. FL, frontal lobe. DKO-A is shown at passage 3 here and for all panels. c, Heatmap displays methylation levels for L1Hs 5' UTRs as measured by Oxford Nanopore sequencing separated into groups by the methylation state. Loess-smoothed regression for methylation levels across full-length L1Hs elements in each group is shown on the right, with the 5' UTR shaded in gray. d. Genome browser tracks show extension of hypomethylation at an unmethylated active ESC enhancer following DNMT3 loss. e, Heatmap of active ESC enhancer methylation levels, in HUES64 WT and DKO-A cells, including $5 \mathrm{~kb}$ on either side. $\mathbf{f}$, Genome browser tracks showing cDKO-DMR overlap with putative tissue-specific enhancers in the frontal lobe. $\mathbf{g}$, Binary heatmap of cDKO-DMR overlap with tissue-specific enhancers. Each row represents the enhancer set of a different tissue or cell type. Rows for H1 ESCs, frontal lobe and CD19+ B cells are indicated. Dark shading indicates overlap with a cDKO-DMR. Hierarchical clustering of rows and columns was performed using the complete linkage method. h, Frontal lobe enhancers separated by their methylation status in WT, DKO-A and the frontal lobe. i, Composite plots (fragments per base pair, per peak) of H3K4me1 and H3K27ac enrichment across cDKO-DMRs in comparison with active ESC (H1) enhancers.

enriched for H3K27me3, indicating absence of a classically 'poised' chromatin state (Extended Data Fig. 5g). We found no enrichment of ESC enhancer-associated transcription factors, though we could confirm that these regions become bound by tissue-specific transcription factors after differentiation (Extended Data Fig. 5h-j). We then expanded our inquiry to other ESC-expressed transcription factors, including eight ChIP-seq datasets from WT HUES64 $\mathrm{ESCs}^{34}$ and 63 ChIP-seq datasets encompassing all Encyclopedia of DNA Elements (ENCODE)-probed H1 ESC factors (Extended Data Fig. 5k,l). Even across this larger transcription factor set we did not find any notable enrichment within CDKO-DMR boundaries, nor did we observe a consistent sequence motif for any known factor (Supplementary Table 2). Currently, we therefore hypothesize that TET recruitment may depend on a transcription factor not included in the above set or an alternative strategy such as via a noncoding RNA tether ${ }^{35}$, or it could involve a more complex regulatory mechanism or interaction that remains to be elucidated.
Finally, we explored whether we could map DKO-DMRs to genes and assess potential regulatory roles. On average, cDKO-DMRs were located $48 \mathrm{~kb}$ from transcription start sites and could be assigned by proximity to 6,594 genes, which does not allow for meaningful pathway or interactome analysis. Instead, we performed RNA sequencing (RNA-seq) to discern whether loss of methylation at these somatic enhancers affects gene expression. Indeed, late-passage DKO cells significantly upregulated genes associated with differentiation $(n=2,455$, false discovery rate $(\mathrm{FDR})<0.01$, fold-change $\geq 2$ ), such as EOMES, SOX17, TGFB2, MSX2, SOX1 and SIX1 (Extended Data Fig. 5m and Supplementary Table 3). Although we cannot distinguish direct from indirect effects, 526 of these genes already showed significant differential expression by passage 6. At passage 28, we found that DKO-DMRs that are associated with a differentially expressed gene are substantially closer to their genes and two-thirds of differentially expressed genes are associated with at least one DKO-DMR (Extended Data Fig. 5m,n). 

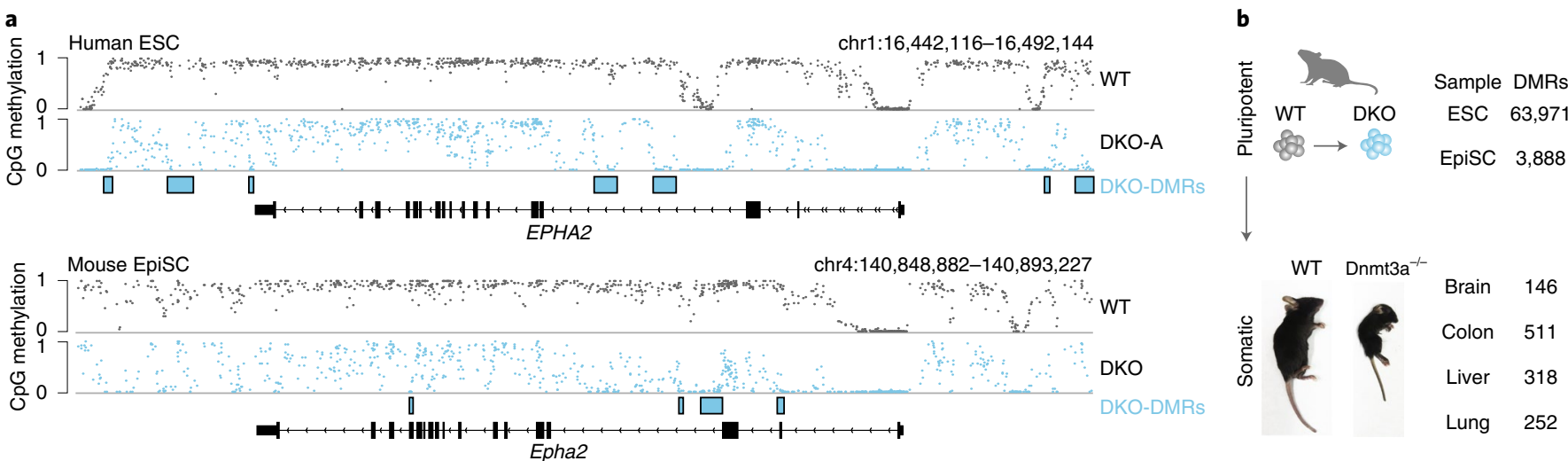

Fig. 4 | Targeted demethylation in the absence of DNMT3 appears conserved in mice. a, Genome browser tracks for an orthologous locus in human ESCs and mouse EpiSCs show the conserved presence of DKO-DMRs following DNMT3 DKO. b, A comparison of DKO-DMRs identified in mouse DKO ESCs, DKO EpiSCs and a range of DKO-like Dnmt3a knockout mouse tissues. The number of DMRs derives from one experiment per condition. Homozygous Dnmt3a knockout mice (-/-) were generated by crossing Dnmt3a heterozygous mice (+/-). A representative photo of a WT and knockout mouse at day 23 is shown to highlight the expected runted phenotype in line with earlier studies ${ }^{14}$. To the right, the number of DMRs identified for each cell type or each tissue is shown.

These trends also support a potential link between demethylation of the targeted regulatory element and upregulated gene expression.

cDKO-DMRs are conserved and associated with pluripotency. To explore the conservation of this locally regulated dynamic methylation, we mutated Dnmt $3 a$ and Dnmt $3 b$ within mouse epiblast stem cells (EpiSCs) as these represent the closest available developmental and molecular analog to human pluripotent cells (Extended Data Fig. 6a,b). We performed WGBS for WT and DKO cells at passage 4 and identified 3,888 DMRs that showed similar properties to human DKO-DMRs, with $82 \%$ overlapping putative somatic enhancers $^{36}$. While the number, precise sequence and co-ordinates did not directly map between species (as is expected for regulatory elements $^{37}$; Extended Data Fig. 6c), mouse DKO-DMRs appeared in remarkably similar locations and in proximity to orthologous genes (Fig. 4a and Extended Data Fig. 6d,e). Thus, both the mechanism of methylation turnover at somatic enhancers and the regulatory logic of these regions appear largely conserved.

As somatic cells also express DNMTs and TETs (Extended Data Fig. 7a,b), we were curious whether the DNMT3/TET interplay at somatic enhancers is exclusive to pluripotency, or whether it is preserved after differentiation. We therefore examined a range of systems that lack DNMT3 activity within various stages of developmental potential (Extended Data Fig. 8). These include mouse ESCs cultured in serum/leukemia inhibitory factor (LIF), which represent a more naïve pluripotent state than EpiSCs. We also examined tissues from homozygous $D n m t 3 a^{-1-}$ mice under the premise that most somatic cells do not express catalytically active DNMT3B (refs. ${ }^{38,39}$ ) and therefore loss of DNMT3A would create 'DKO-like' cells that lack de novo methyltransferase activity (Fig. 4b). We then performed WGBS on WT and DKO ESCs at passage 4 as well as brain, colon, liver and lung tissue from 8-day-old WT and Dnmt $3 a^{-1-}$ mice (Fig. 4b). Compared with EpiSCs, we identified tenfold more DKO-DMRs for ESCs $(n=63,971)$ and tenfold fewer in somatic tissues (brain, 146; colon, 511; liver, 318; lung, 252; Extended Data Fig. 8a-g). The dynamic regulation of cDKO-DMRs therefore appears restricted to pluripotent cell states, with substantially fewer TET-targeted loci as development proceeds. In each case, $73-82 \%$ of the DMRs overlapped with mouse tissue-specific enhancers as defined previously ${ }^{36}$. Somatic Dnmt3a KO DMRs preferentially favor the class 1 subgroup located near CpG islands and shores, suggesting that in this context TETs largely function to preserve the boundary of already unmethylated regions (Extended Data Fig 8h,i).
To complement our mouse data, we reanalyzed WGBS data from HUES64 WT and DNMT3A KO human ESCs differentiated into postmitotic motor neurons ${ }^{40}$. In this system, cells lose protein expression of DNMT3B by day 2 to generate a DKO-like state in viable $3 \mathrm{AKO}$ cells (Extended Data Fig. 8j). Although cells had been without DNMT3 activity for $\sim 12 \mathrm{~d}$, cDKO-DMRs remained highly methylated despite continuous TET1-3 expression, further hinting that the key players involved in this process may be exclusively expressed in the pluripotent state (Extended Data Fig. 8k,l).

TET enzymes also demethylate broadly throughout the genome. After establishing that DNMTs and TETs regulate a subset of somatic enhancers in a pluripotency-specific manner, we returned to the more gradual background demethylation observed in both the DKO and PKO contexts (Extended Data Fig. 2a,c). To avoid confounding our global measurements with the targeted DNMT3 or TET activity described above, we excluded all low-stringency DMRs identified between any pair of samples from further analysis $(n=238,497$; Extended Data Fig. 9a-d), as well as all somatic enhancer regions ${ }^{32}$. Notably, DNMT3 loss in the presence or absence of TETs still resulted in global average methylation decreases of 0.0028 and 0.0037 per passage, respectively (Fig. 5a and Extended Data Fig. 9e,f). Subsequently, we estimate the fidelity of DNA methylation patterns to be 99.75 and 99.64 per cell cycle (estimated using $6 \mathrm{~d}$ per passage and $4 \mathrm{~d}$ per passage, with population doubling time of $28.8 \mathrm{~h}$ and $24 \mathrm{~h}$ for DKO and PKO cells, respectively).

Methylation loss within heterochromatin has been suggested to emerge from low DNMT1 fidelity and mitotic division ${ }^{2}$. In support of this model, we observed preferential loss of methylation within heterochromatic regions for PKO cells that only express DNMT1. In contrast, DKO cells exhibit preferential loss of methylation within euchromatin, implying additional contributions from global TET activity (Fig. 5b and Extended Data Fig. 9g). Upon closer inspection, we find low $5 \mathrm{hmC}$ signal distributed throughout the genome of WT ESCs, including at inert intergenic DNA, that exists at higher levels within euchromatin (Fig. 5b,c). Accordingly, methylation decreases the most in regions with high WT $5 \mathrm{hmC}$ when de novo DNMTs are absent (Fig. 5d,e).

We next differentiated WT HUES64 ESCs to postmitotic motor neurons, where $5 \mathrm{hmC}$ cannot be passively diluted over division, to more specifically track TET engagement and catalytic activity. After $60 \mathrm{~d}$ of culture, $5 \mathrm{hmC}$ levels were enriched within gene bodies (particularly for expressed genes) and intergenic loci, with the greatest 


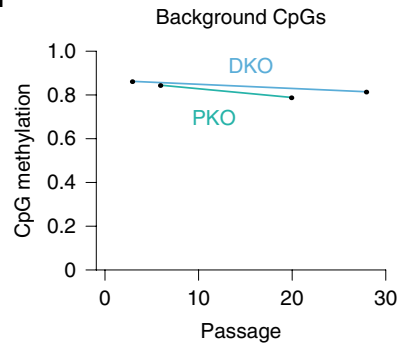

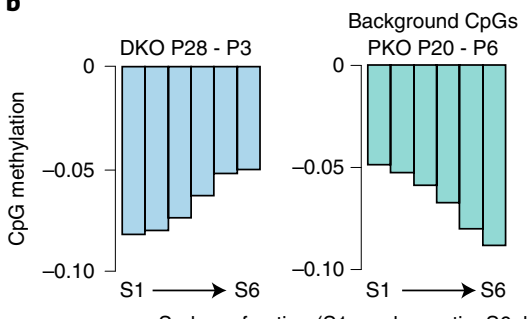

C Background CpGs

HUES64 WT $5 \mathrm{hmC}$

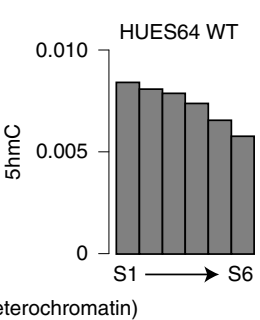

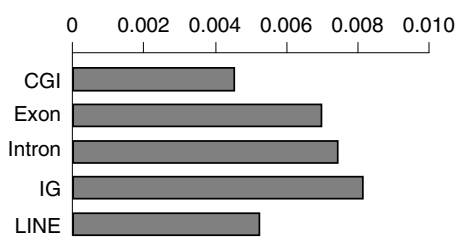

d

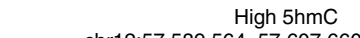

Low $5 \mathrm{hmC}$

$18 \mathrm{~kb} \quad$ chr12:57,676,810-57,702,112

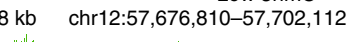

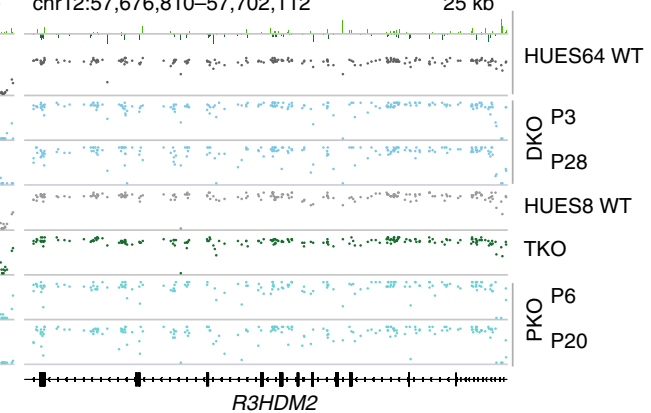

e

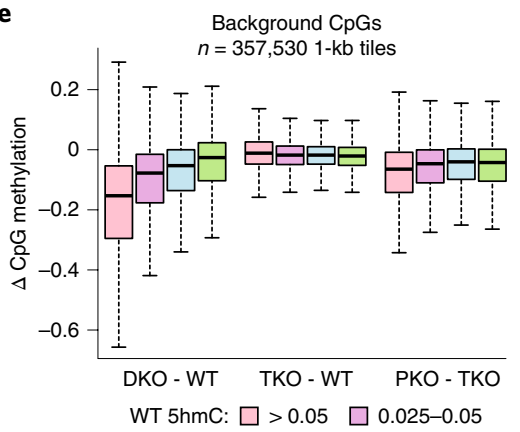

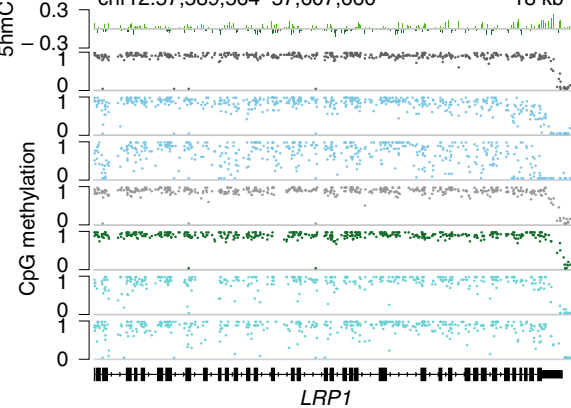

RHDM2

f

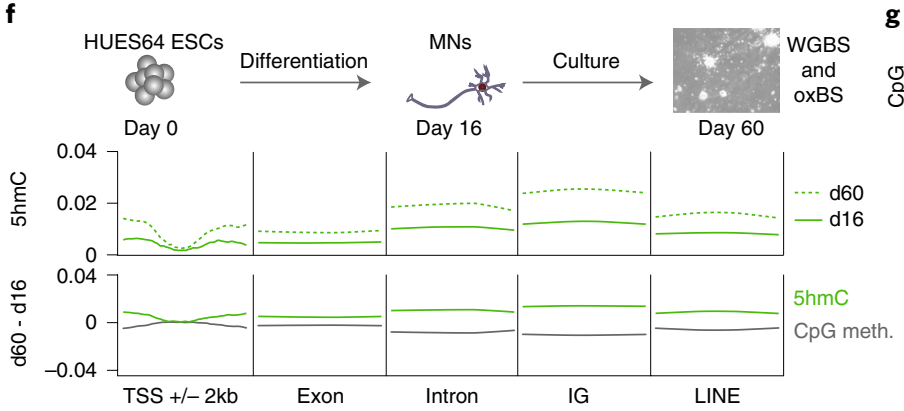

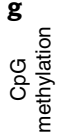

$\square 0.01-0.025 \square<0.01$

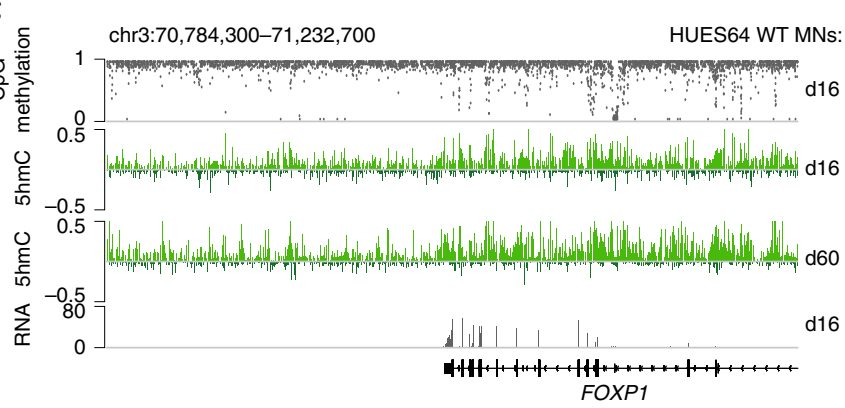

Fig. 5 | TET enzymes also have widespread global activity. a, Mean methylation for matched CpGs $(n=7,905,649)$ in DKO and PKO ESCs. Background $\mathrm{CpGs}$ represent $\mathrm{CpGs}$ outside of defined enhancers or cDKO-DMRs. b. Methylation difference over passaging for DKO and PKO cells (left and center) and WT hydroxymethylcytosine ( $5 \mathrm{hmC}$ ) levels (right). CpGs are separated according to replication timing taken from previous repli-BS-seq data: $\mathrm{S} 1$ is early replicating and enriched for euchromatin, while $\mathrm{S} 6$ is late replicating and enriched for heterochromatin. c, $5 \mathrm{hmC}$ levels in WT cells across different genomic features. d, Genome browser tracks of representative high and low 5hmC enrichment in WT cells. Genomic regions with higher $5 \mathrm{hmC}$ in WT lose more methylation in DKO cells. e, For 1-kb tiles with different mean $5 \mathrm{hmC}$ levels; the difference in methylation between WT and DKO, TKO and WT, or PKO and DKO cells is displayed. Boxes display the interquartile range while the bold line shows the median and whiskers extend to $1.5 \times$ interquartile range. f, Human ESCs were differentiated into motor neurons (MNs) and cultured for $60 \mathrm{~d}$. WGBS and oxBS were performed at day 16 (d16) and d60 and the respective methylation and $5 \mathrm{hmC}$ levels across different genomic features are displayed. $\mathbf{g}$, Genome browser tracks display methylation and $5 \mathrm{hmC}$ levels for MNs at d16 and d60. An increase in 5hmC is observed over the transcribed FOXP1 gene body, but also within the nearby intergenic region.

increase occurring within inert chromatin, supporting widespread oxidation by TETs (Fig. 5f,g and Extended Data Fig. 10a-c). We therefore conclude that TETs maintain the ability to broadly oxidize methylcytosine through the genome of motor neurons as well as ESCs.

Normally, this activity would result in continuous turnover of cytosine modification and a global methylation decrease if not countered by DNMT3A or DNMT3B. Given this paradigm, one might expect ESCs to show a global methylation increase upon TET loss, as this would shift the balance in the favor of DNMT3 activity. Counterintuitively, and as previously noted in cancer cells ${ }^{41}$, a slight decrease was also observed in cells lacking TETs compared with matched WT (Extended Data Fig. 10d). Although speculative, this may be associated with the increased growth rate of TKO cells compared with WT, which could also impact methylation maintenance (Extended Data Fig. 10e).

To understand whether competing activity of DNMTs and TETs can contribute to methylation heterogeneity, as previously suspected for $\mathrm{H} 3 \mathrm{~K} 4 \mathrm{mel}$-enriched enhancers ${ }^{42}$, we performed hairpin bisulfite sequencing to measure symmetrical cytosine methylation on $\mathrm{CpG}$ dyads of the same molecule. We found fewer hemi-methylated dyads in the absence of de novo DNMTs and TETs $(4.48 \%$ in PKO compared with $8.89 \%$ in WT cells), indicating that DNMTs and TETs act together to create epigenetic variation at single loci (Extended Data Fig. 10f-j). The stochastic nature of genome-wide TET activity appears to generate methylation heterogeneity within populations of cells (Extended Data Fig. 10k,l).

\section{Discussion}

Here, we report a comprehensive analysis of a series of human ESC lines devoid of DNMT3 and/or TET activity. We find that TETs are focally recruited to thousands of somatic enhancers that are highly methylated in the pluripotent state, which results in local $5 \mathrm{hmC}$ enrichment and targeted demethylation in the absence of countering DNMT3 activity.

The TET-dependent focal loss of methylation at these somatic elements after the DNMT3s are removed is intriguing for many 
reasons, including its conservation and relative stability. For example, after 28 passages, DNMT3 DKO cells accumulate additional DKO-DMRs, but also preserve the local architecture of immediately demethylated regions. DMRs maintain distinct borders without further erosion, suggesting an initially dynamic switch that is well defined in cis and can be maintained over extended periods. It remains to be determined what factors are responsible for coordinating TET recruitment in ESCs. We found that the three TET enzymes can act redundantly and appear to find their targets independently of the TET CXXC domain. Although motif as well as preliminary transcription factor binding enrichment analyses could not elucidate a clear candidate recruiting factor, we cannot rule out potential transcription factor-mediated recruitment in general as many factors are yet to be interrogated by ChIP-seq in ESCs and have unknown binding motifs. Whatever the ultimate mechanism, it is most likely to be pluripotency-associated, as we observed no such loss of methylation at these loci in somatic cells similarly depleted of DNMT3 activity. It is also possible that there is no specific recruitment factor. Previous work shows that all three TETs can be ubiquitinated by CLR4, which is predicted to induce a conformational change that promotes direct TET binding to $\mathrm{DNA}^{43,44}$. It is therefore also possible that post-translational modification or unknown mechanisms may regulate TET recruitment or activity at certain target sites. Proteomics and biochemical strategies may be useful to explore these additional possibilities.

The focal competition between DNMTs and TETs at enhancers with somatic functions highlights a distinct epigenetically regulated state within pluripotent cells. Around one-third of somatic enhancers are known to attract analogous competing activities, but are instead kept in an unmethylated state in ESCs and are instructed by histone modifications ${ }^{45-47}$. Here, we now describe that another third remain highly methylated despite a similar recruitment of DNMT3s and TETs. For this set, which can be identified only by DNMT3 knockout, the central question becomes why thousands of somatic enhancers actively recruit TET enzymes despite a default methylated state.

We hypothesize that this strategy could facilitate rapid switching of methylation states that may be required to eliminate this modification efficiently during early cell fate transitions. For instance, active regulation may support rapid changes in cell cycle-dependent control that are required for the exit from pluripotency towards the early germ layers ${ }^{48,49}$. Simultaneously, these activities would also enable robust activation upon exposure to the appropriate differentiation signals. Based on our limited expression analysis, we speculate that keeping these regions methylated in pluripotent cells may be important to prevent binding of ESC-expressed or early developmental transcription factors that would otherwise lead to unwanted expression of differentiation-associated genes. Conversely, methylated or hydroxymethylated cytosines may support binding of certain transcription factors ${ }^{50}$. Carefully designed experiments will be required to further tease these possible models apart and assign biological importance of these sites.

We further note that the pluripotent-specific nature of DNA methylation-based enhancer regulation found in our study suggests that some epigenetic states may be more robustly controlled within certain developmental windows than in others. Counterintuitively, the dynamic interplay of opposing DNMT and TET activities at these sites may ensure that they are more faithfully maintained during a period where they might otherwise influence the developmental outcome. In this context, it will be interesting to explore whether the dynamics observed for LINEs represents a hijacked feature of somatic enhancer regulation to trigger activation during pluripotency-related stages, such as the germline, where DNMT3 activity is acutely downregulated. This early shift in genomic regulation would allow temporal L1Hs upregulation before alternative means of suppression (such as by H3K27 methylation ${ }^{51}$ ). Such cooption of an endogenous, pluripotency-specific mechanism would be analogous to what has been described for endogenous retroviruses ${ }^{52,53}$.

We also observe genome-wide activity of DNMT3s and TETs that causes a methylation shift after their loss. At this broader scale, we found increased TET-dependent DNA demethylation within euchromatin, which intuitively suggests that methylated cytosine located in open chromatin is more amenable to stochastic oxidation. It seems reasonable that targeted TET activity at DKO-DMRs is desirable, while global demethylation represents an unavoidable off-target activity due to the proximity of enzymes to their substrate. Of course, background oxidation comes at a high cost that requires continuous de novo activity to maintain a highly methylated mammalian genome. From this view, a better understanding of these counteracting forces will be also relevant to understand various disease states. For example, DNMT3A is frequently mutated in cancer $^{54}$ and impaired in several neurodegenerative diseases ${ }^{55}$, two somatic contexts where catalytic DNMT3B is not expressed and cells presumably lack all de novo activity.

Taken together, our results highlight focal competition between opposing DNA-methylating and -demethylating enzymes that uncovers another unique layer of epigenetic regulation within pluripotent cells.

\section{Online content}

Any methods, additional references, Nature Research reporting summaries, source data, extended data, supplementary information, acknowledgements, peer review information; details of author contributions and competing interests; and statements of data and code availability are available at https://doi.org/10.1038/ s41588-020-0639-9.

Received: 7 March 2019; Accepted: 30 April 2020;

Published online: 8 June 2020

\section{References}

1. Jadhav, U. et al. Extensive recovery of embryonic enhancer and gene memory stored in hypomethylated enhancer DNA. Mol. Cell 74, 542-554.e545 (2019).

2. Zhou, W. et al. DNA methylation loss in late-replicating domains is linked to mitotic cell division. Nat. Genet. 50, 591-602 (2018).

3. Jin, Z. \& Liu, Y. DNA methylation in human diseases. Genes Dis. 5, 1-8 (2018).

4. Klutstein, M., Nejman, D., Greenfield, R. \& Cedar, H. DNA methylation in cancer and aging. Cancer Res. 76, 3446-3450 (2016).

5. Gangisetty, O., Cabrera, M. A. \& Murugan, S. Impact of epigenetics in aging and age related neurodegenerative diseases. Front. Biosci. (Landmark Ed.) 23, 1445-1464 (2018).

6. Ziller, M. J. et al. Charting a dynamic DNA methylation landscape of the human genome. Nature 500, 477-481 (2013).

7. Tahiliani, M. et al. Conversion of 5-methylcytosine to 5 -hydroxymethylcytosine in mammalian DNA by MLL partner TET1. Science 324, 930-935 (2009).

8. Ito, S. et al. Role of Tet proteins in $5 \mathrm{mC}$ to $5 \mathrm{hmC}$ conversion, ES-cell self-renewal and inner cell mass specification. Nature 466, 1129-1133 (2010).

9. Ito, S. et al. Tet proteins can convert 5-methylcytosine to 5-formylcytosine and 5-carboxylcytosine. Science 333, 1300-1303 (2011).

10. He, Y. F. et al. Tet-mediated formation of 5-carboxylcytosine and its excision by TDG in mammalian DNA. Science 333, 1303-1307 (2011).

11. Maiti, A. \& Drohat, A. C. Thymine DNA glycosylase can rapidly excise 5-formylcytosine and 5-carboxylcytosine: potential implications for active demethylation of CpG sites. J. Biol. Chem. 286, 35334-35338 (2011).

12. Smith, Z. D. \& Meissner, A. DNA methylation: roles in mammalian development. Nat. Rev. Genet. 14, 204-220 (2013).

13. Li, E., Bestor, T. H. \& Jaenisch, R. Targeted mutation of the DNA methyltransferase gene results in embryonic lethality. Cell 69, 915-926 (1992).

14. Okano, M., Bell, D. W., Haber, D. A. \& Li, E. DNA methyltransferases Dnmt3a and Dnmt3b are essential for de novo methylation and mammalian development. Cell 99, 247-257 (1999).

15. $\mathrm{Gu}, \mathrm{T}$. P. et al. The role of Tet3 DNA dioxygenase in epigenetic reprogramming by oocytes. Nature 477, 606-610 (2011).

16. Dawlaty, M. M. et al. Combined deficiency of Tet 1 and Tet 2 causes epigenetic abnormalities but is compatible with postnatal development. Dev. Cell 24, 310-323 (2013). 
17. Baubec, T. et al. Genomic profiling of DNA methyltransferases reveals a role for DNMT3B in genic methylation. Nature 520, 243-247 (2015).

18. Liao, J. et al. Targeted disruption of DNMT1, DNMT3A and DNMT3B in human embryonic stem cells. Nat. Genet. 47, 469-478 (2015).

19. Manzo, M. et al. Isoform-specific localization of DNMT3A regulates DNA methylation fidelity at bivalent CpG islands. EMBO J. 36, 3421-3434 (2017)

20. Jeong, M. et al. Large conserved domains of low DNA methylation maintained by Dnmt3a. Nat. Genet. 46, 17-23 (2014).

21. Verma, N. et al. TET proteins safeguard bivalent promoters from de novo methylation in human embryonic stem cells. Nat. Genet. 50, 83-95 (2018).

22. $\mathrm{Wu}, \mathrm{H}$. et al. Dual functions of Tetl in transcriptional regulation in mouse embryonic stem cells. Nature 473, 389-393 (2011).

23. Lu, F., Liu, Y., Jiang, L., Yamaguchi, S. \& Zhang, Y. Role of Tet proteins in enhancer activity and telomere elongation. Genes Dev. 28, 2103-2119 (2014).

24. Wang, L. et al. TET2 coactivates gene expression through demethylation of enhancers. Sci. Adv. 4, eaau6986 (2018).

25. Hon, GaryC. et al. $5 \mathrm{mC}$ oxidation by Tet 2 modulates enhancer activity and timing of transcriptome reprogramming during differentiation. Mol. Cell 56 286-297 (2014).

26. Huang, Y. et al. Distinct roles of the methylcytosine oxidases Tet1 and Tet2 in mouse embryonic stem cells. Proc. Natl Acad. Sci. USA 111, 1361-1366 (2014).

27. $\mathrm{Xu}$, Y. et al. Genome-wide regulation of $5 \mathrm{hmC}, 5 \mathrm{mC}$, and gene expression by Tet1 hydroxylase in mouse embryonic stem Cells. Mol. Cell 42, 451-464 (2011).

28. Ko, M. et al. Modulation of TET2 expression and 5-methylcytosine oxidation by the CXXC domain protein IDAX. Nature 497, 122-126 (2013).

29. Zhang, W. et al. Isoform switch of TET1 regulates DNA demethylation and mouse development. Mol. Cell 64, 1062-1073 (2016).

30. de la Rica, L. et al. TET-dependent regulation of retrotransposable elements in mouse embryonic stem cells. Genome Biol. 17, 234 (2016).

31. Rinaldi, L. et al. Dnmt3a and Dnmt3b associate with enhancers to regulate human epidermal stem cell homeostasis. Cell Stem Cell 19, 491-501 (2016).

32. Hnisz, D. et al. Super-enhancers in the control of cell identity and disease. Cell 155, 934-947 (2013).

33. Alvarez-Dominguez, J. R. et al. Circadian entrainment triggers maturation of human in vitro islets. Cell Stem Cell 26, 108-122.e110 (2020).

34. Tsankov, A. M. et al. Transcription factor binding dynamics during human ES cell differentiation. Nature 518, 344-349 (2015).

35. Guallar, D. et al. RNA-dependent chromatin targeting of TET2 for endogenous retrovirus control in pluripotent stem cells. Nat. Genet. 50, 443-451 (2018).

36. Shen, Y. et al. A map of the cis-regulatory sequences in the mouse genome. Nature 488, 116-220 (2012).

37. Cheng, Y. et al. Principles of regulatory information conservation between mouse and human. Nature 515, 371-375 (2014).

38. Huntriss, J. et al. Expression of mRNAs for DNA methyltransferases and methyl-CpG-binding proteins in the human female germ line, preimplantation embryos, and embryonic stem cells. Mol. Reprod. Dev. 67, 323-336 (2004)

39. Gordon, C. A., Hartono, S. R. \& Chédin, F. Inactive DNMT3B splice variants modulate de novo DNA methylation. PLoS ONE 8, e69486 (2013).

40. Ziller, M. J. et al. Dissecting the functional consequences of de novo DNA methylation dynamics in human motor neuron differentiation and physiology. Cell Stem Cell 22, 559-574.e559 (2018).

41. López-Moyado, I. F. et al. Paradoxical association of TET loss of function with genome-wide DNA hypomethylation. Proc. Natl Acad. Sci. USA 116, 16933-16942 (2019)

42. Rulands, S. et al. Genome-scale oscillations in DNA methylation during exit from pluripotency. Cell Syst. 7, 63-76.e12 (2018).

43. Nakagawa, T. et al. CRL4(VprBP) E3 ligase promotes monoubiquitylation and chromatin binding of TET dioxygenases. Mol. Cell 57, 247-260 (2015).

44. Yu, C. et al. CRL4 complex regulates mammalian oocyte survival and reprogramming by activation of TET proteins. Science 342, 1518-1521 (2013).

45. Creyghton, M. P. et al. Histone H3K27ac separates active from poised enhancers and predicts developmental state. Proc. Natl Acad. Sci. USA 107, 21931-21936 (2010).

46. Nguyen, M. L. T., Jones, S. A., Prier, J. E. \& Russ, B. E. Transcriptional enhancers in the regulation of T cell differentiation. Front. Immunol. 6 462 https://doi.org/10.3389/fimmu.2015.00462 (2015).

47. Rada-Iglesias, A. et al. A unique chromatin signature uncovers early developmental enhancers in humans. Nature 470, 279-283 (2010).

48. Pauklin, S., Madrigal, P., Bertero, A. \& Vallier, L. Initiation of stem cell differentiation involves cell cycle-dependent regulation of developmental genes by cyclin D. Genes Dev. 30, 421-433 (2016).

49. Yiangou, L. et al. Cell cycle regulators control mesoderm specification in human pluripotent stem cells. J. Biol. Chem. 294, 17903-17914 (2019).

50. Yin, Y. et al. Impact of cytosine methylation on DNA binding specificities of human transcription factors. Science 356, eaaj2239 (2017).

51. Walter, M., Teissandier, A., Pérez-Palacios, R. \& Bourc'his, D. An epigenetic switch ensures transposon repression upon dynamic loss of DNA methylation in embryonic stem cells. eLife 5, el1418 (2016).

52. Kunarso, G. et al. Transposable elements have rewired the core regulatory network of human embryonic stem cells. Nat. Genet. 42, 631-634 (2010).

53. Schmidt, D. et al. Waves of retrotransposon expansion remodel genome organization and CTCF binding in multiple mammalian lineages. Cell 148, 335-348 (2012).

54. Spencer, D. H. et al. CpG island hypermethylation mediated by DNMT3A is a consequence of AML progression. Cell 168, 801-816.e813 (2017).

55. Cirulli, E. T. et al. Exome sequencing in amyotrophic lateral sclerosis identifies risk genes and pathways. Science 347, 1436-1441 (2015).

Publisher's note Springer Nature remains neutral with regard to jurisdictional claims in published maps and institutional affiliations.

(c) The Author(s), under exclusive licence to Springer Nature America, Inc. 2020 


\section{Methods}

We have complied with all relevant ethical regulations. All human ESC work was approved by Harvard University's Embryonic Stem Cell Research Oversight (ESCRO) and the mouse work by the Institutional Animal Care and Use Committee (IACUC; protocol no. 28-21).

Cell culture. Human ESCs were grown in feeder-free conditions using Geltrex (Thermo Fisher Scientific) and mTeSR (STEMCELL Technologies) and split every $4-5 \mathrm{~d}$ using StemPro Accutase Cell Dissociation Reagent (Thermo Fisher Scientific). Mouse ESCs were cultured on mitomycin-C-treated mouse embryonic fibroblasts (MEFs) in standard conditions (knockout DMEM containing 15\% fetal bovine serum, $100 \mu \mathrm{M}$ nonessential amino acids, $1 \mathrm{mM}$ L-glutamine, $1 \times$ penicillin-streptomycin, $0.1 \mathrm{mM} 2$-mercaptoethanol and $10^{3}$ units of ESGRO LIF) and split every $3 \mathrm{~d}$ using TrypLE (Thermo Fisher Scientific). E3 GOF18-EGFP male EpiSCs (a generous gift from the Schöler laboratory) were cultured feeder-free as described previously ${ }^{56}$ (MEF-conditioned medium consisting of knockout DMEM supplemented with $20 \%$ knockout serum replacement, $100 \mu \mathrm{M}$ nonessential amino acids, $1 \mathrm{mM}$ L-glutamine, $1 \times$ penicillin-streptomycin, $10 \mathrm{ng} \mathrm{ul}^{-1} \mathrm{FGF} 2,0.1 \mathrm{mM}$ 2 -mercaptoethanol) and were split into clumps every $3 \mathrm{~d}$ using collagenase type IV (STEMCELL Technologies). All lines were regularly tested and found negative for Mycoplasma contamination.

Generation of Dnmt3a/3b DKO ESCs and EpiSCs. To create Dnmt3a and Dnmt3b DKO mouse ESCs and EpiSCs, we targeted both genes simultaneously by transfecting the cells with px458 (Addgene plasmid no. 48138) containing guides targeting the highly conserved PC motif in the catalytic domains in ESCs, and guides targeting both the PC motif as well as early coding exons in EpiSCs (Supplementary Table 4 ). Knockouts were verified by genotyping and western blot. Mouse ESCs were transfected using the Amaxa 4D nucleofector X-Unit (Lonza) according to the manufacturer's guidelines, and EpiSCs were transfected using Xfect Transfection Reagent (Clontech) according to the manufacturer's guidelines.

Nanopore sequencing and data processing. Ultra-high-molecular-weight genomic DNA (gDNA) was extracted from cells using a phenol-chloroform extraction. gDNA was then sheared to an average length of $20-\mathrm{kb}$ fragments using g-TUBEs (Covaris) following the manufacturer's recommendations. Sheared gDNA was then size-selected using the BluePippin High Pass Plus Gel Cassette (Sage Science). Sheared and purified gDNA was then used to prepare Nanopore libraries using the Ligation Sequencing Kit 1D (SQK-LSK108) following the manufacturer's guidelines. All MinION sequencing runs were processed using the Nanopype pipeline $^{57}$ (v.0.9.0). The basecaller Guppy (v.3.1.5) was used with the r9.4.1 fast configuration. Quality filtering was disabled for any basecalling. Alignments were made against human genome hg38 using $\operatorname{NGMLR}^{58}$ (v.0.2.7) with the ont parameter preset. Based on the same alignments, genome-wide methylation tracks were obtained from nanopolish (v.0.11.0) $)^{59}$ and structural variations were called using Sniffles ${ }^{58}$ (v.1.0.10). Nanopolish was used with default parameters while filtering for $\mathrm{CpGs}$ with $>5 \times$ coverage. Sniffles was configured to report variations of at least $500 \mathrm{bp}$, supported by at least five reads from read fragments longer than $2 \mathrm{~kb}(-1500-\mathrm{s} 5-\mathrm{r} 2000)$. From the Sniffles output, L1Hs elements were identified as homozygous, heterozygous or missing, and only those that were homozygous and at least $6 \mathrm{~kb}$ in length were used for subsequent analysis.

Generation of HUES8 mutant lines. PKO cells were obtained by disrupting the DNMT3A catalytic domain (exon 19) in HUES8 QKO cells using CRISPRCas9 (sgRNA listed in Supplementary Table 4). The sgRNA was cloned into the pSPCas9n(BB)-GFP (Addgene px458) vector and transfected into HUES8 QKO cells with FuGene HD (Promega) followed by sorting for green fluorescent protein (eGFP) signal for selection. After 10-14 d of culture, single-cell clones were divided in a 1:3 ratio for maintenance and validation of gene editing, respectively.

HUES8 DNMT3A $A^{--} ;$DNMT3B $B^{-/}$DKO mutants were derived by first targeting DNMT3A using the same method and sgRNA described for PKO cells above. After confirming the DNMT3A knockout, the DNMT3B catalytic domain (exon 19) was targeted using three different DNMT3B gRNAs (Supplementary Table 4) cloned into the px458 vector and transiently transfected into the HUES8

$D N M T 3 A^{-/-}$cells. To assess correct knockout mutants, the PCR fragments around each sgRNA target region from individual clones were subcloned into pCR Blunt II-TOPO vector (Invitrogen), followed by Sanger sequencing with M13 primers or sequencing primers. In addition, western blot analyses were performed with whole-cell lysate.

TET PiggyBac plasmid construction. The coding sequences for FLAG-TET2-P2A-GFP (GenScript, custom synthesis of TET2 (NM_001127208.2) in pcDNA3.1(+)-P2A-eGFP vector) and FLAG-HA-TET3 (Addgene no. 49446) were excised from their plasmids by restriction digest of $5 \mu \mathrm{g}$ of plasmid with ApaI and AflII, or KpnI-HF and XbaI, respectively (all NEB, $1 \mu$ of each was digested at $37^{\circ} \mathrm{C}$ for $120 \mathrm{~min}$ ). Subsequently, the corresponding fragments were gel-purified (Qiagen).

The target vector pPiggyBac MCS (a modified version of plasmid no. SPB007, Transposagen, with additional cloning sites) was digested in the same manner and additionally dephosphorylated (NEB, $1 \mu \mathrm{l}$ of $\mathrm{AP}, 37^{\circ} \mathrm{C}$ for $20 \mathrm{~min}$ ) before gel purification. The coding sequences were cloned into pPiggyBac MCS using the Quick Ligation kit (NEB) in a ratio of 1:3 and transformed into Escherichia coli (NEB stbl TET2, DH5 $\alpha$ (Meissner laboratory) for TET3). Plasmids were isolated (Qiagen) and the correct sequence of the constructs was confirmed by Sanger sequencing.

The T2A-BFP sequence was obtained from pU6-sgRNA EF1Alpha-puro-T2A-BFP (Addgene no. 60955) by PCR amplification using Gibson primers with overhangs for pPiggyBac-TET3 digested with XbaI (Supplementary Table 4). After gel purification, the T2A-BFP amplicon was inserted into pPiggyBac-TET3 using the NEBuilder HiFi DNA Assembly Master Mix (NEB) in a ratio of $1: 5\left(50^{\circ} \mathrm{C}, 30 \mathrm{~min}\right)$ and transformed into E. coli (DH5 $\alpha$; Meissner laboratory). Plasmids were isolated and the correct sequence of the constructs was confirmed by Sanger sequencing.

The TET1 coding sequence was obtained by PCR amplification of pIRES-hrGFP II-TET1 (Addgene no. 83568) using Gibson primers to create overhangs for pPiggyBac MCS digested with XbaI (Supplementary Table 4). As we wanted the shorter TET1 isoform, the primers amplify only the necessary sequence and introduce an additional ATG. After gel purification, the TET1 amplicon was inserted into pPiggyBac MCS using the NEBuilder HiFi DNA Assembly Master Mix (NEB) in a ratio of 1:5 and transformed into E. coli (DH5 $\alpha$; Meissner laboratory). Plasmids were isolated and the correct sequence of the constructs was confirmed by Sanger sequencing. One of the confirmed clones of pPiggyBac TET1 was then digested with XbaI and assembled with the P2A-mCherry amplicon (obtained by PCR amplification from pDnmt3b1-P2A-mCherry ${ }^{60}$ ) using the NEBuilder HiFi DNA Assembly Master Mix (NEB) in a ratio of 1:5 and transformed into E. coli (DH5 $\alpha$; Meissner laboratory). Plasmids were isolated and the correct sequence of the constructs was confirmed by Sanger sequencing.

TET rescue experiments. TET1, TET2 or TET3 transposons described above were introduced using the PiggyBac system, by cotransfection with a plasmid encoding the transposase (TransposagenBio). We used a 3:1 molar ratio of transposon to transposase, transfecting $70 \%$ confluent PKO cells using the Lipofectamine 3000 reagent (Invitrogen) according to the manufacturer's instructions. After overnight incubation of the DNA complex on the cells, the cell monolayers were washed with PBS and incubated with mTeSR-1 containing RevitaCell supplement (Gibco) for a further $2 \mathrm{~d}$. Successful integration of each TET enzyme in PKO cells was assessed by FACS, and positive cells were placed back into culture for expansion. About 5-7 weeks after transfection, positive cells were collected by FACS followed by snap-freezing of the cell pellets. For a control, we transfected $70 \%$ confluent PKO cells with the transposase only. These cells were also sorted and plated in low density to mirror the rescue experiments.

Hairpin bisulfite sequencing. WT and PKO ESCs were arrested in G2/M phase with $2 \mu \mathrm{g} \mathrm{ml}^{-1}$ Nocodazole for $16 \mathrm{~h}$. Accutase (STEMCELL Technologies) was used to detach cells, which were then snap-frozen before gDNA extraction using the Quick-DNA universal kit (Zymo Research). Following this, $3 \mu \mathrm{g}$ of DNA was sheared to average fragment size of $200 \mathrm{bp}$ using Covaris Microtube and S2 ultrasonicator (Covaris). After shearing, DNA was concentrated to $50 \mu \mathrm{l}$ in water with 1.8 volumes of Agencourt AMPure XP beads (Beckman Coulter) and was prepared for Illumina sequencing using Kapa LTP library preparation kit (Kapa Biosystems). We modified a previously described hairpin adapter for bisulfite sequencing ${ }^{61}$ by including a 12-bp unique molecular identifier with methylated cytosine bases (Integrated DNA Technologies; Supplementary Table 4). Barcoded fork-adapters for Illumina sequencing (NEXTflex bisulfite-seq barcodes) were purchased from Bioo Scientific. After postligation purification with 0.7 volumes of AMPure XP beads, biotinylated DNA molecules were pulled down on $50 \mu \mathrm{l}$ of Dynabeads MyOne Streptavidin C1 beads (Thermo Fisher Scientific). Libraries were detached from $\mathrm{C} 1$ beads by heating them in $20 \mu \mathrm{l}$ of $10 \mathrm{mM}$ Tris pH 7.5 buffer at $95^{\circ} \mathrm{C}$ for $10 \mathrm{~min}$. After separating beads on a magnet, DNA in supernatant was bisulfite-converted using the EpiTect Fast Bisulfite Kit (Qiagen), extending both $60^{\circ} \mathrm{C}$ incubation steps to $20 \mathrm{~min}$ each. DNA was then subjected to 20 cycles of amplification using primers provided with Bioo Scientific adapters and Kapa Uracil+ amplification master mix (Kapa Biosystems). Amplified DNA was loaded on $2 \%$ precast gel E-gelEX (Thermo Fisher Scientific) and fragments of size 500-650 bp were selected by gel extraction using Zymoclean Gel DNA recovery kit (Zymo Research). Final libraries were sequenced with 300-base paired-end reads on the Illumina Miseq platform. Fastqs were aligned to the hg19 human genome, then reads were filtered to eliminate those with identical barcodes. Next, we used HBS-tools ${ }^{62}$ for adapter trimming and methylation dyad calling.

Western blot in human cell lines. Western blot analysis was performed with whole-cell lysate. The cell monolayer was rinsed three times with ice-cold PBS before collecting cells with a cell lifter (VWR) and pelleting by centrifugation. The cell pellets were lysed in $20 \mathrm{mM}$ HEPES pH 8.0, $100 \mathrm{mM} \mathrm{NaCl}, 1 \mathrm{mM}$ EDTA and $1 \%$ Tween 20 containing protease inhibitor cocktail (ROCHE) on ice for $30 \mathrm{~min}$ and then sonicated, and cell debris was cleared by centrifugation. The protein concentration of the supernatant was quantified using the Micro BCA Protein Assay Kit (Pierce). About 70-100 $\mu$ g of total protein was separated on 4-12\% 
SDS-PAGE gel (Invitrogen) and transferred to PVDF membranes (GE Healthcare), followed by incubation with anti-rabbit-DNMT3A antibody (Abcam ab188470 lot:GR224165-13) and anti-rabbit-GAPDH antibody (Cell Signaling 2118 lot: 10) as a loading control. All blots were developed using the ECL Reaction Kit (Bio-Rad) according to the manufacturer's instructions.

Western blot in mouse cell lines. Cells were collected and washed twice in PBS before being resuspended in cell lysis buffer $\left(25 \mathrm{mM}\right.$ HEPES pH 7.6, $5 \mathrm{mM} \mathrm{MgCl}_{2}$, $25 \mathrm{mM} \mathrm{KCl}, 0.05 \mathrm{mM}$ EDTA, $10 \%$ glycerol, $0.1 \%$ IGEPAL, $1 \times$ Roche protease inhibitor, $1 \mathrm{mM}$ dithiothreitol). The cell lysate was spun for $5 \mathrm{~min}$ at 1,500 r.p.m. to pellet the nuclei. Nuclei were washed once to remove cell debris $(10 \mathrm{mM}$ HEPES pH 7.6, $3 \mathrm{mM} \mathrm{MgCl}, 100 \mathrm{mM} \mathrm{KCl}, 0.01 \mathrm{mM}$ EDTA, $10 \%$ glycerol, $1 \times$ Roche protease inhibitor, $1 \mathrm{mM}$ dithiothreitol). Nuclei were spun down at $3,000 \mathrm{~g}$ for $5 \mathrm{~min}$. Nuclei were then resuspended in $150 \mu \mathrm{l}$ of RIPA buffer and vortexed for $20 \mathrm{~min}$ at $4{ }^{\circ} \mathrm{C}$. This mixture was spun at 12,000 r.p.m. for $15 \mathrm{~min}$ and the supernatant was collected. Then, $20 \mu \mathrm{l}$ of $4 \times$ LDS buffer was added to $60 \mu \mathrm{l}$ of supernatant and denatured at $72{ }^{\circ} \mathrm{C}$ for $10 \mathrm{~min}$. Western blots were performed with anti-Dnmt1 (1:1,000 dilution, Abcam ab87654 lot: GR3194562-7), anti-Dnmt3a (1:2,000, Abcam ab188470 lot: GR224165-2), anti-Dnmt3b (1:500, Abcam ab176166 lot: GR3199224-3), anti-histone H3 (Abcam ab1791 lot: GR293197-1) and anti-GAPDH (Cell Signaling Technology mAb no. 2118L lot: 10) antibodies and imaged using HRP chemiluminescence.

Dnmt3a knockout mice. As Dnmt3a knockout mice die at around 4 weeks after birth $^{14}$, we crossed heterozygous Dnmt3a knockout mice in which the Dnmt3a catalytic domain, PC and ENV motifs were replaced with a cassette containing an IRES-lacZ gene and a neomycin-resistance gene (B6;129S4-Dnmt3a $<$ tm1Enl $>$ ) to obtain homozygous offspring. At $8 \mathrm{~d}$ after birth, we collected colon, lung and liver tissue for WGBS. Mice were genotyped using primers specific for the WT or mutant locus (Supplementary Table 4).

Motor neuron differentiation and culture. HUES64 WT human ESCs were cultured in mTeSR medium (STEMCELL Technologies), which was replenished daily. Once $>75 \%$ confluence had been reached, colonies were dissociated using Accutase (Innovative Cell Technologies). Human ESCs were then maintained in mTeSR with $10 \mu \mathrm{M}$ ROCK inhibitor (Y-27632, DNSK International) for $24 \mathrm{~h}$ before being cultured in mTeSR alone. At $>70 \%$ confluence, differentiation was initiated. For $14 \mathrm{~d}$, cells were maintained in a 1:1 ratio of Neurobasal and DMEM/ F12 medium supplemented with N2, B27, GlutaMax and nonessential amino acids (N2B27; Thermo Fisher, Life Technologies). Additionally, medium contained $1 \mu \mathrm{M}$ retinoic acid (Sigma) and $1 \mu \mathrm{M}$ smoothened agonist (DNSK International). For the first $6 \mathrm{~d}$, cells were administered dual-SMAD inhibitors SB-431542 (10 $\mu \mathrm{M}$, DNSK International) and LDN-193189 (100 nM, DNSK International). For the remaining $8 \mathrm{~d}$, cells were treated with $5 \mu \mathrm{M}$ Notch inhibitor DAPT (DNSK International) and $4 \mu \mathrm{M}$ VEGF/FGF inhibitor SU5402 (DNSK International). Medium with small molecules was refreshed daily. At day 14, cells were dissociated using TrypLE Express with DNase I (Thermo Fisher, Worthington) and plated at a density of 400,000 cells per well in a 12-well plate. Neurons were maintained in Neurobasal medium supplemented with N2, B27, GlutaMax and nonessential amino acids. Medium was additionally supplemented with neurotrophic factors BDNF, CNTF, GDNF (10 $\mathrm{ng} \mathrm{ml}^{-1}, \mathrm{R} \& D$ systems) and AA ( $200 \mathrm{ng} \mathrm{ml}^{-1}$, Sigma). For the first $24 \mathrm{~h}$, neurons were maintained in $2 \%$ Hyclone FBS (VWR) with Y-27632 ROCK inhibitor $\mathrm{EdU}(10 \mu \mathrm{M}$, Thermo Fisher) was administered from day 14 to 21 . Half of the volume of medium with small molecules was refreshed every $2-3 \mathrm{~d}$. For collection, neurons were washed twice with PBS and gently lifted from the wells using a cell scraper. Neurons were then pelleted and snap-frozen until sample processing.

WGBS. DNA was extracted using the Zymo Quick-DNA Universal Kit, and bisulfite conversion was carried out using the Qiagen Epitect Fast kit, both according to the manufacturer's instructions. The eluted DNA was processed immediately using the Accel-NGS Methyl-seq DNA library kit (Cat. no. DL-ILMMS-12, Swift Biosciences) following the manufacturer's recommendations. PCR products were cleaned up using the Agencourt AMPure XP system (Beckman Coulter cat. no. A63881). The absence of adapter-dimers was confirmed using an Agilent Bioanalyzer. We then sequenced the libraries on the HiSeq4000, NovaSeq S2 or Hiseq2500, generating 150-base paired-end reads.

Whole-genome oxBS. gDNA from HUES64 cells was extracted using the Quick-DNA universal kit (Zymo Research). First, 400 ng of DNA was sheared to average fragment size of $200 \mathrm{bp}$ using S2 Focused-ultrasonicator (Covaris). After shearing, DNA was concentrated to $50 \mu \mathrm{l}$ in water with 1.8 volumes of Agencourt AMPure XP beads (Beckman Coulter) and was processed with TrueMethyl Whole Genome kit (Cambridge Epigenetix, now available from Tecan) in hydroxymethylation mode up to the library preparation step. The library construction module was replaced with the Accel-NGS Methyl-Seq kit (Swift Biosciences) following the manufacturer's recommendations.

WGBS/oxBS data processing. Raw sequencing reads were trimmed using cutadapt and aligned against the human (hg19) or mouse (mm9) genome using
BSMAP. Duplicate reads were removed using Picard tools. Methylation calls were made using MOABS mCall module and only CpGs covered with at least ten reads were considered for further analysis. To generate $5 \mathrm{hmC}$ levels, the methylation state derived from WGBS $(\mathrm{mC}+5 \mathrm{hmC})$ was subtracted from the methylation state derived from oxBS (mC) for CpGs with coverage of at least ten reads.

Data analysis. For comparison with genomic features, BedTools was used to intersect methylation data within $\mathrm{CpG}$ islands ${ }^{63}$, coding features (RefSeq), H1 ESC-defined typical and super enhancers ${ }^{32}$, and repeats (RepeatMasker).

Chromatin modification peaks or BAM files were taken from ENCODE (H1 ESCs) and used to compare overlap or local enrichment. We also used all available BAM files from ChIP-seq transcription factor experiments performed in $\mathrm{H} 1$ cells to study the enrichment at cDKO-DMRs. Here, we used the software Homer ${ }^{64}$ to create tag directories for each bam file, then used Homer findMotifsGenome.pl to determine the enrichment at features of interest.

Violin plots, box plots, heatmaps, composite plots and scatter plots were all created in R. IGV was used to visualize CpG-specific methylation levels throughout the genome and create browser images.

RNA-seq. RNA-seq libraries were prepared using the TrueSeq RNA Sample Prep v2 HS Protocol (Illumina), followed by 50 -base paired-end sequencing on the NextSeq500 Sequencing System (Illumina). RNA-seq data were aligned using STAR aligner followed by stringtie v.1.3 to the hg 19 genome. $\log _{2}$ TPM (transcripts per million) values were used to plot expression levels in R.

Statistics. For WGBS data analysis, to identify discrete regions regulated by TETs or DNMTs, we performed DMR calling by using a CpG-specific $F$-test. For stringent DMRs, we merged regions where at least $8 / 10 \mathrm{CpGs}$ had $P<0.01$ with a difference of $\geq 0.6$, and for reduced stringency we used $5 / 8$ CpGs with $P<0.01$ and difference $\geq 0.25$. DMRs were merged if they were located within $1 \mathrm{~kb}$ of each other. After calling per sample DKO-DMRs in our three clones HUES64 DKO-A, DKO-B and HUES8 DKO, we identified $n=17,684,19,715$ and 20,456 DKO-DMRs, respectively. We then merged these into one region list (causing overall DMRs per sample to decrease in the Venn diagram seen in Fig. 1d). From this list, a region was determined as demethylated if the mean methylation level was below 0.2. Consensus cDKO-DMRs had mean methylation below 0.2 in all three samples.

To compare mouse Dnmt3a ${ }^{-/}$DKO-DMRs, all tissue-specific DKO-DMRs were combined, then a region was considered demethylated if the difference between WT and Dnmt $3 \mathrm{a}^{-/-}$was at least 0.2 .

For RNA-seq data analysis, the R package edgeR was used. 'glmQLFit' was used to fit a quasi-likelihood negative binomial generalized log-linear model to count data, and then 'glmQLFTest' was used to calculate $P$ values and Bonferroni-corrected FDR values for each gene. Genes with FDR $<0.01$ and a $\log$ fold-change of at least 2 were selected as differentially expressed. We also filtered out genes where both groups had $\log$ count values $<2.5$, which was considered as no change in expression even though this can derive highly significant $P$ values.

All $t$-tests were two-sided. Note that the software R outputs the minimal $P$ value $2.2 \times 10^{-16}$, so where stated, the true $P$ value may in fact be smaller.

Reporting Summary. Further information on research design is available in the Nature Research Reporting Summary linked to this article.

\section{Data availability}

Data have been deposited in the Gene Expression Omnibus under accession GSE126958. Other published datasets used in this study include: HUES64 DNMT3A single-knockout ESCs (GSM1545005), DNMT3B single-knockout ESCs (GSM1545006) and mouse WT ESCs (GSM2339908). Source data for Extended Data Figs. 1,6 and 8 are provided.

\section{References}

56. Greber, B. et al. Conserved and divergent roles of FGF signaling in mouse epiblast stem cells and human embryonic stem cells. Cell Stem Cell 6 215-226 (2010)

57. Giesselmann, P., Hetzel, S., Müller, F.-J., Meissner, A. \& Kretzmer, H. Nanopype: a modular and scalable nanopore data processing pipeline. Bioinformatics 35, 4536 (2019).

58. Sedlazeck, F. J. et al. Accurate detection of complex structural variations using single-molecule sequencing. Nat. Methods 15, 461-468 (2018)

59. Simpson, J. T. et al. Detecting DNA cytosine methylation using nanopore sequencing. Nat. Methods 14, 407-410 (2017).

60. Galonska, C. et al. Genome-wide tracking of dCas9-methyltransferase footprints. Nat. Commun. 9, 597 (2018). 
61. Zhao, L. et al. The dynamics of DNA methylation fidelity during mouse embryonic stem cell self-renewal and differentiation. Genome Res. 24, 1296-1307 (2014).

62. Sun, M.-A., Velmurugan, K. R., Keimig, D. \& Xie, H. HBS-tools for hairpin bisulfite sequencing data processing and analysis. Adv. Bioinformatics 2015, 760423-760423 (2015)

63. Illingworth, R. et al. A novel CpG island set identifies tissue-specific methylation at developmental gene loci. PLoS Biol. 6, e22 (2008).

64. Heinz, S. et al. Simple combinations of lineage-determining transcription factors prime cis-regulatory elements required for macrophage and B cell identities. Mol. Cell 38, 576-589 (2010).

\section{Acknowledgements}

We thank members of the Meissner laboratory for their support, in particular $\mathrm{H}$.

Kretzmer and S. Grosswendt for critical reading of the manuscript as well as R. Karnik for initial processing of the mouse Dnmt3a knockout data. We also thank D. Huangfu and her team for providing the TKO and QKO HUES8 cells. We also acknowledge the Max Planck Institute for Molecular Genetics Sequencing Core. E.K. is a Les Turner ALS Research Center Investigator and a New York Stem Cell Foundation-Robertson Investigator, and part of this work was supported by NIH grant no. NINDS/NIA R01NS104219. A.G. and A.M. are supported by NIGMS grant no. P01GM099117 and A.M. is supported by the Max Planck Society.

\section{Author contributions}

J.C. and A.M. designed the study. J.C. performed data processing and bioinformatic analyses. E.J.J. generated the human ESC knockouts, cultured cells and collected samples. A.L.M. generated the mouse ESC and EpiSC knockouts and performed Nanopore sequencing together with B.B. J.L. generated the Dnmt3a knockout mice. E.J.M. and E.K. differentiated, cultured and collected human motor neurons. N.B. generated the TET rescue constructs. E.K.S. Z.D.S. and A.G. performed library preparation and sequencing. P.G. processed the Nanopore data and F.-J.M. cosupervised the Nanopore work. J.C., Z.D.S. and A.M. interpreted the data and wrote the manuscript.

\section{Competing interests}

The authors declare no competing interests.

\section{Additional information}

Extended data is available for this paper at https://doi.org/10.1038/s41588-020-0639-9. Supplementary information is available for this paper at https://doi.org/10.1038/ s41588-020-0639-9.

Correspondence and requests for materials should be addressed to A.M.

Reprints and permissions information is available at www.nature.com/reprints. 
a

DNMT3A: bottom strand $=$ coding, 634-912aa $=$ catalytic domain, full length $=$ 912aa hg38, chr2:25,240,355-25,240,412

WT

$$
\begin{aligned}
& + \text { Cr CAAAGAGCCAGAAGAAGGGGCGAICAICICCCICCII-GGGCGCGCATCATGCAG } \\
& \text { - GAGTTTCTCGGTCTTCTTCCCCGCTAGTAGAG GGAA-CCCGGCGCGTAGIACGTC }
\end{aligned}
$$

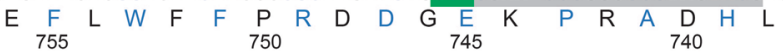

HUES8 DKO + CTCAAAGAGCCAGAAGAAGGGGCGATCATCTCCCTCCTTTGGGCCGCGCATCATGCAG Homozygous - GAGTTTCTCGGTCTTCTTCCCCGCTAGTAGAGGGAGGAAACCCGGCGCGTAGTACGTC

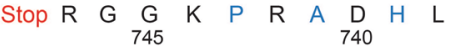

$\begin{array}{lrl}\text { HUES64 DKO } & + \text { CTCAAAGAG } \\ \text { Clone A } & - \text { GAGTTCTC- } & \\ \text { Homozygous } & \text { Stop L A } & \text { D H L }\end{array}$

HUES64 DKO

Clone B

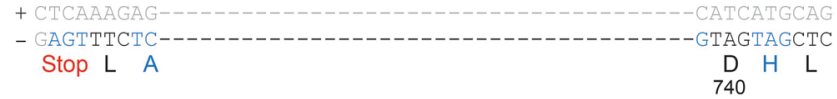

PKO + CTCAAAGAGCCAGAAGAAGGGGCGATCATCTCCCTCCTTTGGGCCGCGCATCATGCAG Homozygous - GAGTTICTCGGTCTTCTTCCCCGCTAGTAGAGGGAGGAAACCCGGCGCGTAGTACGTC Stop R G $\underset{745}{G}$ K $\quad P \quad R \quad A \underset{740}{D} \quad H \quad L$

b

DNMT3B: top strand $=$ coding, $575-853 \mathrm{aa}=$ catalytic domain, full length $=853$ aa hg38, chr20:32,801,301-32,801,360

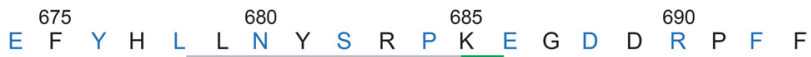

WT + GAATTTACCACCTGCTGAATTACTCACGCCCCAAG AGGGTGATGACCGGCCGTTCTTC

$$
\text { E } \stackrel{675}{\mathrm{~F}} \text { Y } \mathrm{H} \quad \mathrm{L} \quad \mathrm{L} \stackrel{680}{\mathrm{~N}}
$$
Stop

HUES8 DKO GAATTTTACCACCTGCI N Homozygous FTCLCTGATT-

hg38, chr20:32,805,338-32,805,416

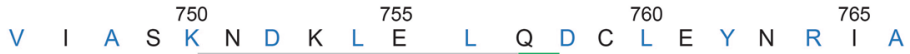

WT

$$
\text { + GTG-ATAGCATCAAAGAATGATAAACTCGAG--CT-GCAG ACTGCTTGGAATACAATAGGATAGCC }
$$

- CAC-TATCGTAGTTTCTTACTATTTGAGCTC--GA-CGTCCTGACGAACCTTATGTTATCCTATCGG

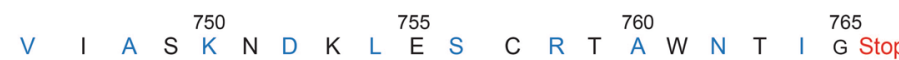

HUES64 DKO + GTG-ATAGCATCAAAGAATGATAAACTCGAGTCCT-GCAGGACTGCTTGGAATACAATAGGATAGCC Clone A - CAC-TATCGTAGTTCTTACTATTTGAGCTCAGGA-CGTCCTGACGAACCTTATGTTATCCTATCGG Homozygous

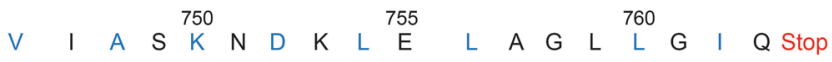

HUES64 DKO + GTG-ATAGCATCAAAGAATGATAAACTCGAG--CTTGCAGGACTGCTTGGAATACAATAGGATAGCC Clone B - CAC-TATCGTAGTTTCTTACTATTTGAGCTC--GAACGTCCTGACGAACCTTCTGTTATCCTATCGG Homozygous

$$
\begin{array}{ll} 
& \text { V N S I K E Stop } \\
\text { PKO } & + \text { GTGAATAGCATCAAAGAATGATAAATCGAG--CT-GCAGGACTGCTTGGAATACAATAGGATAGCC } \\
\text { Homozygous } & \text { - CACTTATCGTAGTTTCTTACTATTTGAGCTC--GA-CGTCCTGACGAACCTTATGTTATCCTATCGG }
\end{array}
$$

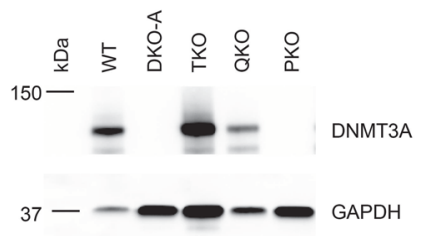

d
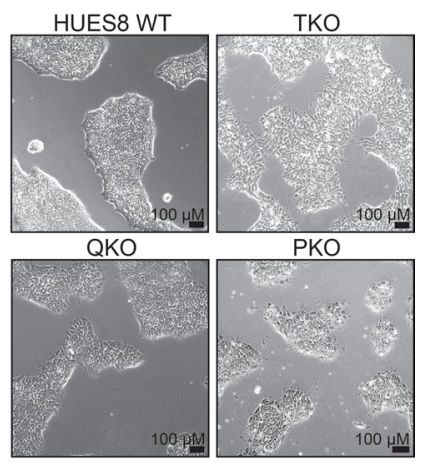

e

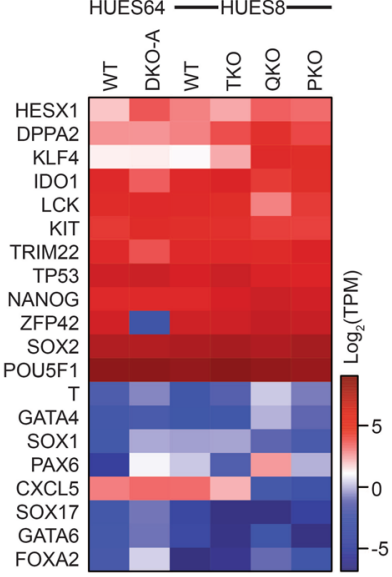

f

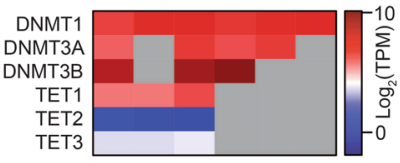

g

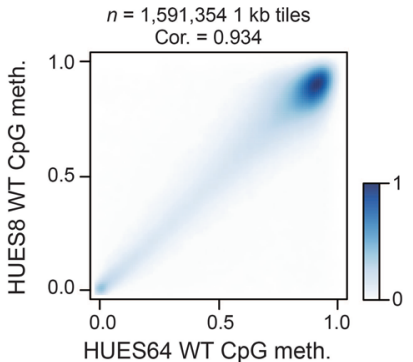

Extended Data Fig. 1 | See next page for caption. 
Extended Data Fig. 1 | Characterization of pentuple knockout ESCs. a, Sequencing data showing the mutations introduced into the DKO and PKO ESCs for DNMT3A. Green box = PAM sequence, grey box = sgRNA sequence. Codons are colored black or blue, grey text denotes non-coding DNA. The respective amino acid (aa) single letter name and sequence number is displayed below the DNA. b, Sequencing data for DNMT3B, as described in a. A different sgRNA was used to target DNMT3B in HUES8 ESCs compared to HUES64. c, Western blot for DNMT3A in HUES8 WT, HUES64 DKO clone A, HUES8 TKO, QKO and PKO cells. GAPDH is shown as the loading control. Full blots in Source Data Fig. 1. PKO cells were assessed for DNMT3A expression by Western blot six times with consistent results. d, HUES8 lines at 10X magnification showing that KO cell lines appear morphologically normal. These images are representative of a full $10 \mathrm{~cm}$ culture dish over weeks of passaging. e, Heatmap showing RNA sequencing data as $\log _{2}$ TPM expression values for a set of pluripotency and self-renewal associated genes (highly expressed), as well as a subset of differentiation associated genes (low expression). DKO-A was collected at passage 6 and PKO at passage 12. f. Heatmap showing RNA sequencing data as $\log _{2}$ TPM expression values DNMTs and TETs, grey boxes depict genes that are knocked out in each sample. $\mathbf{g}$, Comparison of global methylation levels between HUES64 and HUES8 wildtype cells where intensity of blue shading indicates density of data points. The Pearson correlation coefficient (cor.) is displayed. 
a

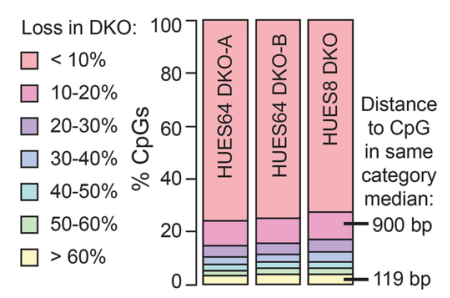

b

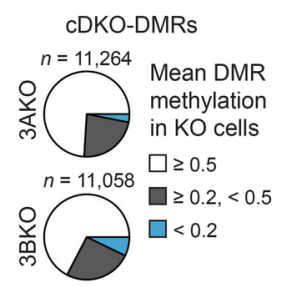

C

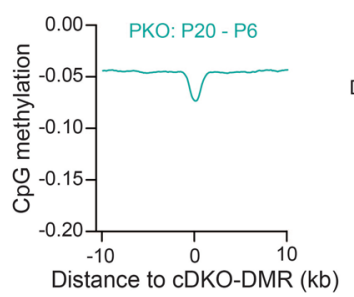

d

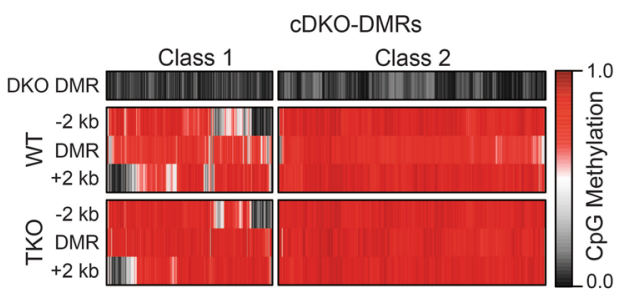

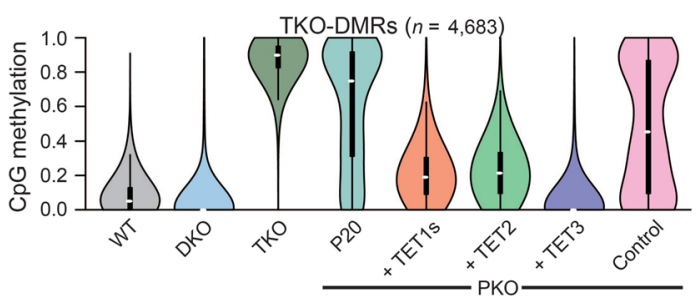

f

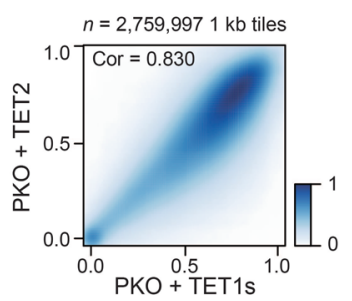

g

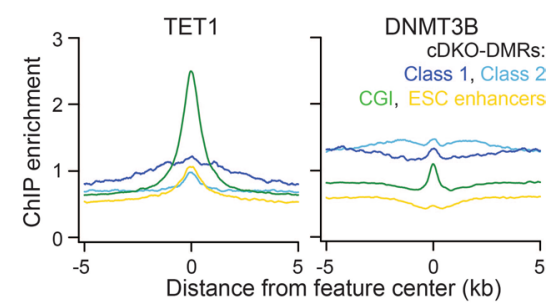

h

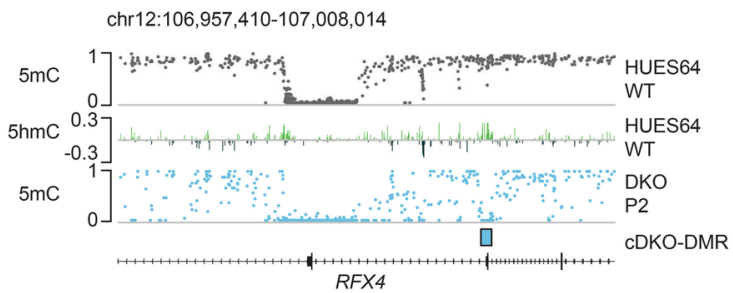

Extended Data Fig. 2 | Characterization of DKO-DMRs and TET rescue in PKO cells. a, Bar chart showing the proportion of CpGs with varying methylation loss in DKO cells compared to WT. CpGs that lost $>60 \%$ methylation were generally located in closer proximity to one another compared to $\mathrm{CpGs}$ that lost $10-20 \%$ methylation.b. The proportion of cDKO-DMRs with respective mean methylation levels in single DNMT3A ${ }^{-/-}$(3AKO) or $D_{N M T 3 B^{-1-}}(3 B K O)$ knockout ESCs. c, Composite plot showing methylation difference between passage (P) 20 and 6 in PKO cells as distance from the CDKO-DMR center. There is a small background loss $(-0.045)$ with a slightly greater focal decrease $(-0.07)$, but methylation levels stay generally high. d, The methylation level in HUES64 DKO-A, HUES64 WT and HUES8 TKO cells including 2 kb from each boundary. For regions where the neighboring 2 $\mathrm{kb}$ is hypomethylated in WT cells (class 1), almost every region shows an increase in methylation following TET loss. e, Violin plots showing methylation at TKO-DMRs across different HUES8 lines. We used stringent parameters to define TKO-DMRs that aberrantly gain methylation upon loss of TET expression (also described later in Extended Data Fig. 9a). Methylation levels were rescued by re-expression of TET. Violin plots extend from the data minima to the maxima, white dot indicates median, thick bar shows the interquartile range and thin bar shows $1.5 x$ interquartile range. $\mathbf{f}$, Methylation levels across $1 \mathrm{~kb}$ tiles for PKO ESCs rescued with either TET1s or TET2. Intensity of blue shading indicates the relative density of data points. Pearson correlation coefficient (cor) is displayed. $\mathbf{g}$, ChIP-seq (fragments per bp, per peak) enrichment (from ref. ${ }^{21}$ ) for TET1 and DNMT3B over CpG islands (CGI), H1-specific enhancers and CDKO-DMRs. h, Representative genome browser tracks displaying methylation levels in WT and DKO cells as well as $5 \mathrm{hmC}$ levels in WT ESCs. Increased 5hmC is observed over the CDKO-DMR and at the border of the hypomethylated CGI, where TETs are known to localize. 
a

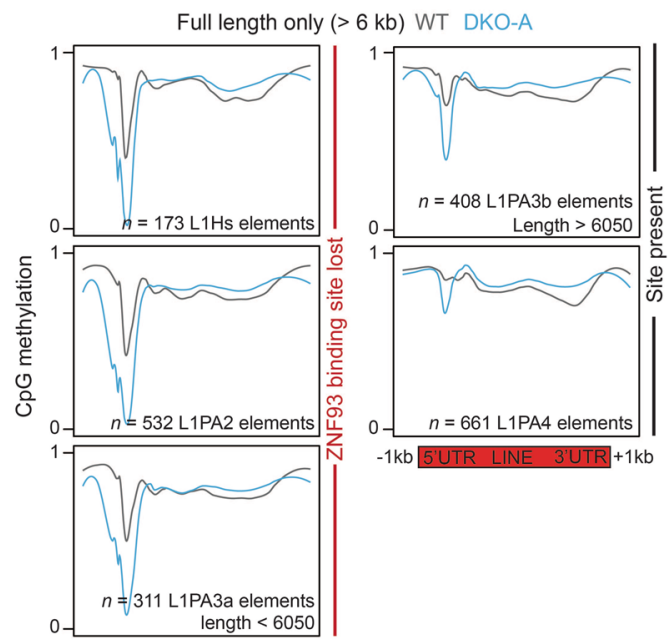

b

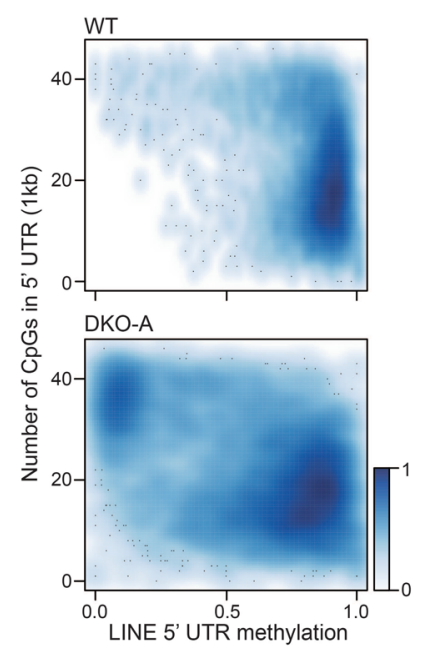

C

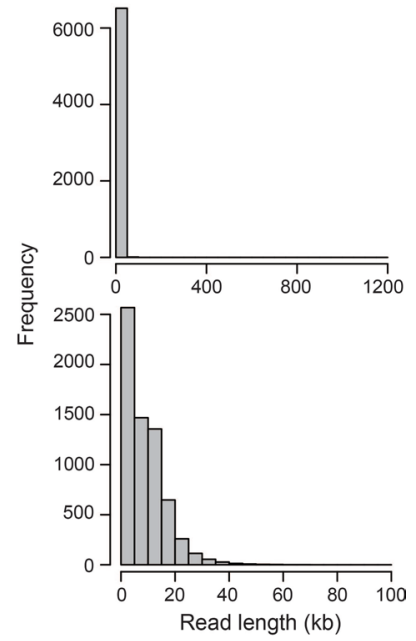

d
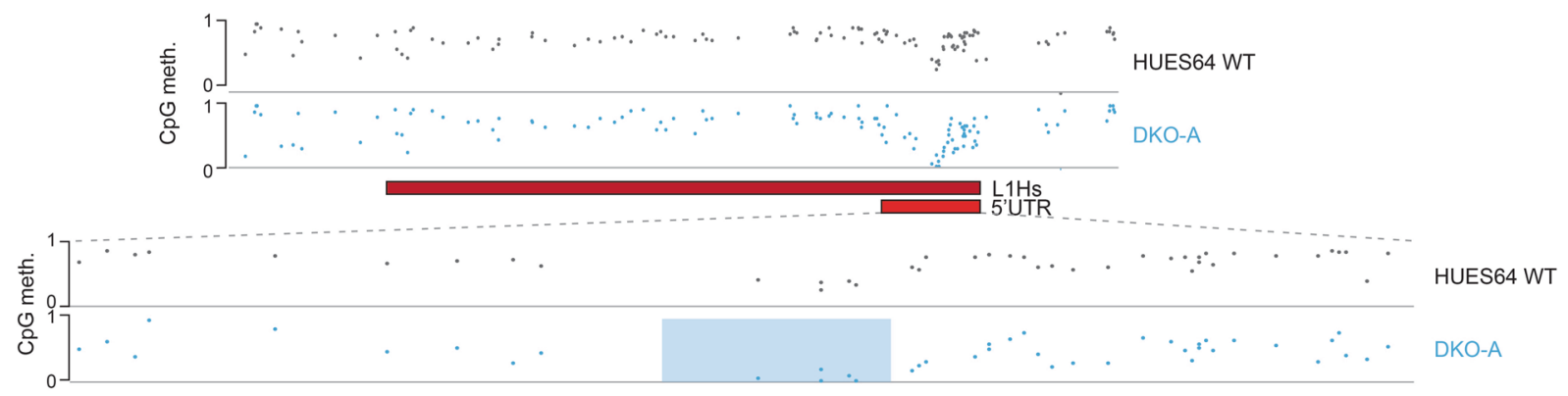

e

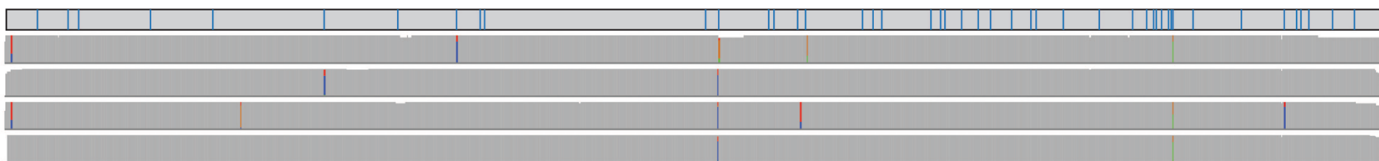

CpG location Group 1: DKO high Group 2: PKO lost Group 3: WT hypo Group 4: DKO only
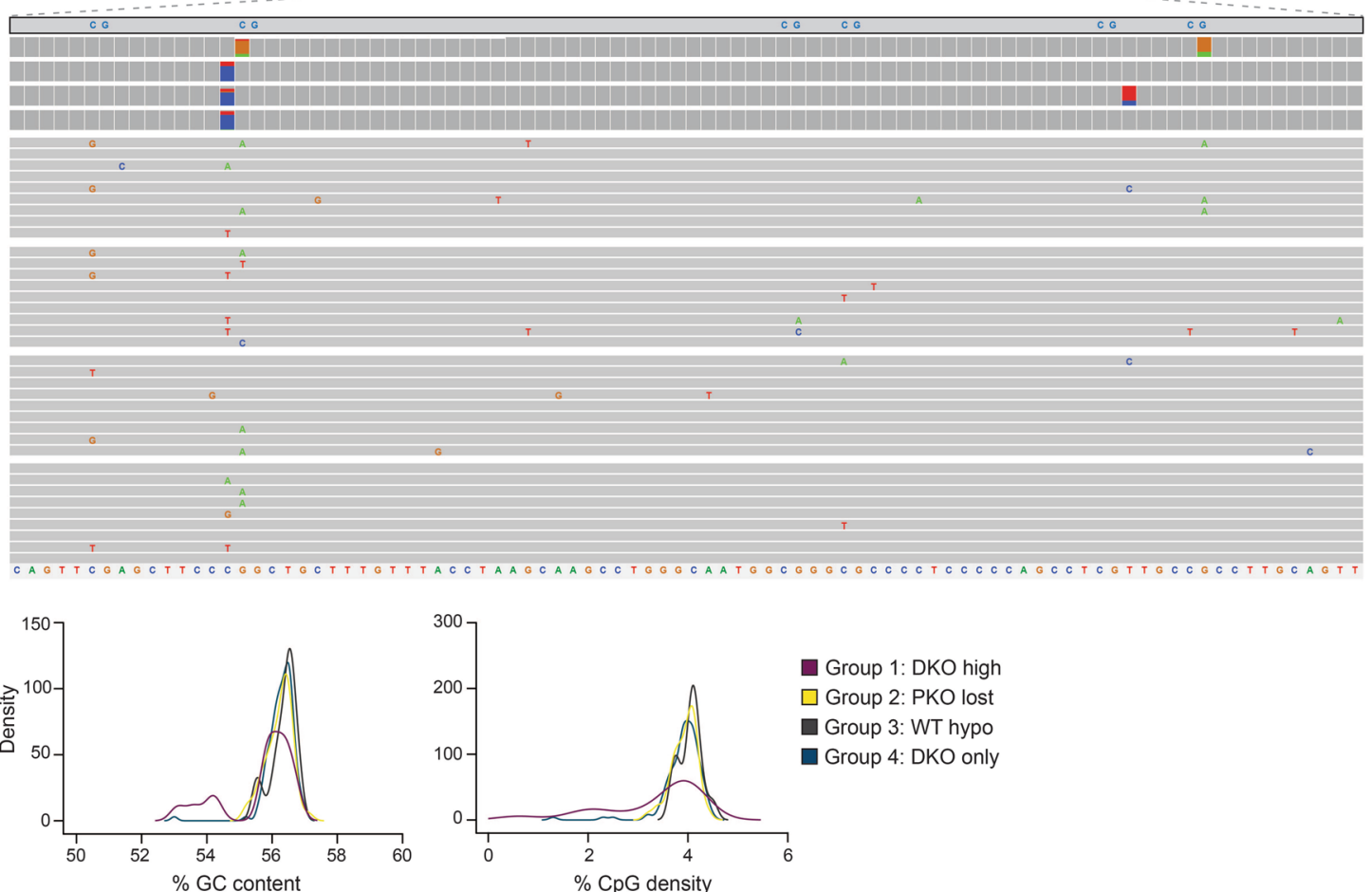

Group 1: DKO high Group 2: PKO lost Group 3: WT hypo Group 4: DKO only

Group 1: DKO high Group 2: PKO lost A

Group 3: WT hypo

Group 4: DKO only

f

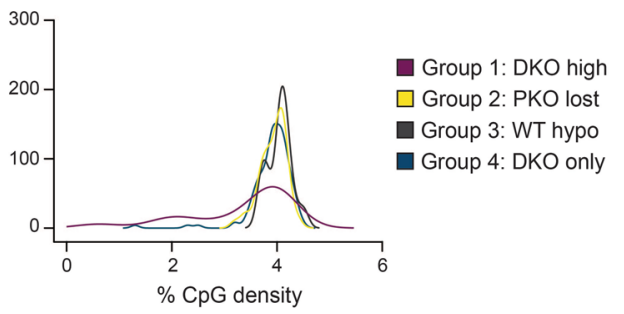

Extended Data Fig. 3 | See next page for caption. 
Extended Data Fig. 3 | LINE 5'UTRs are actively demethylated. a, Methylation composite plots for WT and DKO-A cells over LINE L1 elements (oriented with 5'UTR to the left). L1Hs are the youngest and L1PA4 the oldest class displayed. 5'UTR methylation of younger elements appears more sensitive to DNMT3 loss. In particular, sensitivity appears associated with ZNF93 binding site loss within the $5^{\prime}$ UTR. b. Smoothed scatter plots display the relationship between WT or DKO methylation level and CpG density within the $5^{\prime} U T R$, for all LINE L1 elements where intensity of blue shading indicates density of data points. Lower CpG density elements tend to retain methylation after DNMT3 loss while CpG dense 5'UTRs lose methylation. $\mathbf{c}$, Histogram of read lengths generated from Nanopore sequencing for HUES64 WT cells. Top = all reads, bottom = reads $<100 \mathrm{~kb}$. $\mathbf{d}$, Example of CpG methylation across a group 4 $\mathrm{L} 1 \mathrm{H}$ s element (defined in Figure 3c) showing the focal decrease within the 5'UTR. Below, just the 5'UTR is displayed and CpGs with the most methylation loss following DNMT3 knockout are highlighted with a blue box. e, Each L1Hs 5'UTR was aligned to the consensus sequence. The CpG locations within this sequence are illustrated by vertical blue lines. Below, a summary for genetic variants at each position is shown for each group of L1Hs. Colored boxes display the proportion of bases that vary from the consensus sequence. For each group, nine example $5^{\prime} U T R$ sequences are displayed for the region containing the six $\mathrm{CpGs}$ highlighted in $\mathrm{d}$. Each element is represented as a grey bar with colored letters indicating variations from the consensus sequence (shown at the bottom of the panel). None of the groups exhibit a unique common variant. $\mathbf{f}$, Density plots showing the distribution of $5^{\prime} \mathrm{UTR}$ GC content and $\mathrm{CpG}$ density for each group of L1Hs. 
a

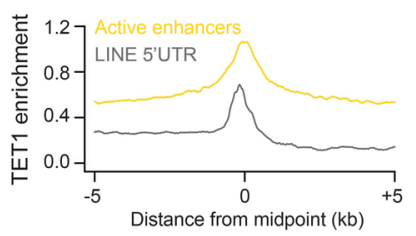

C

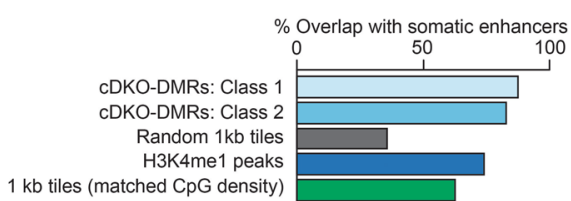

d

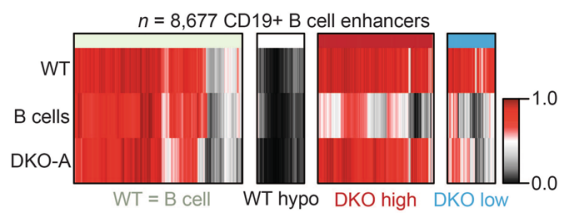

e

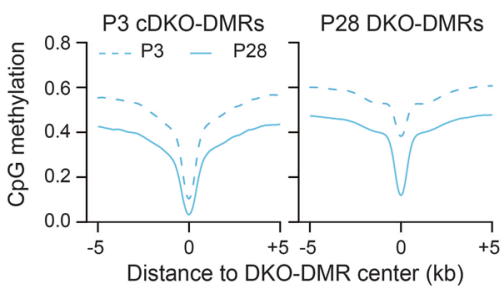

b
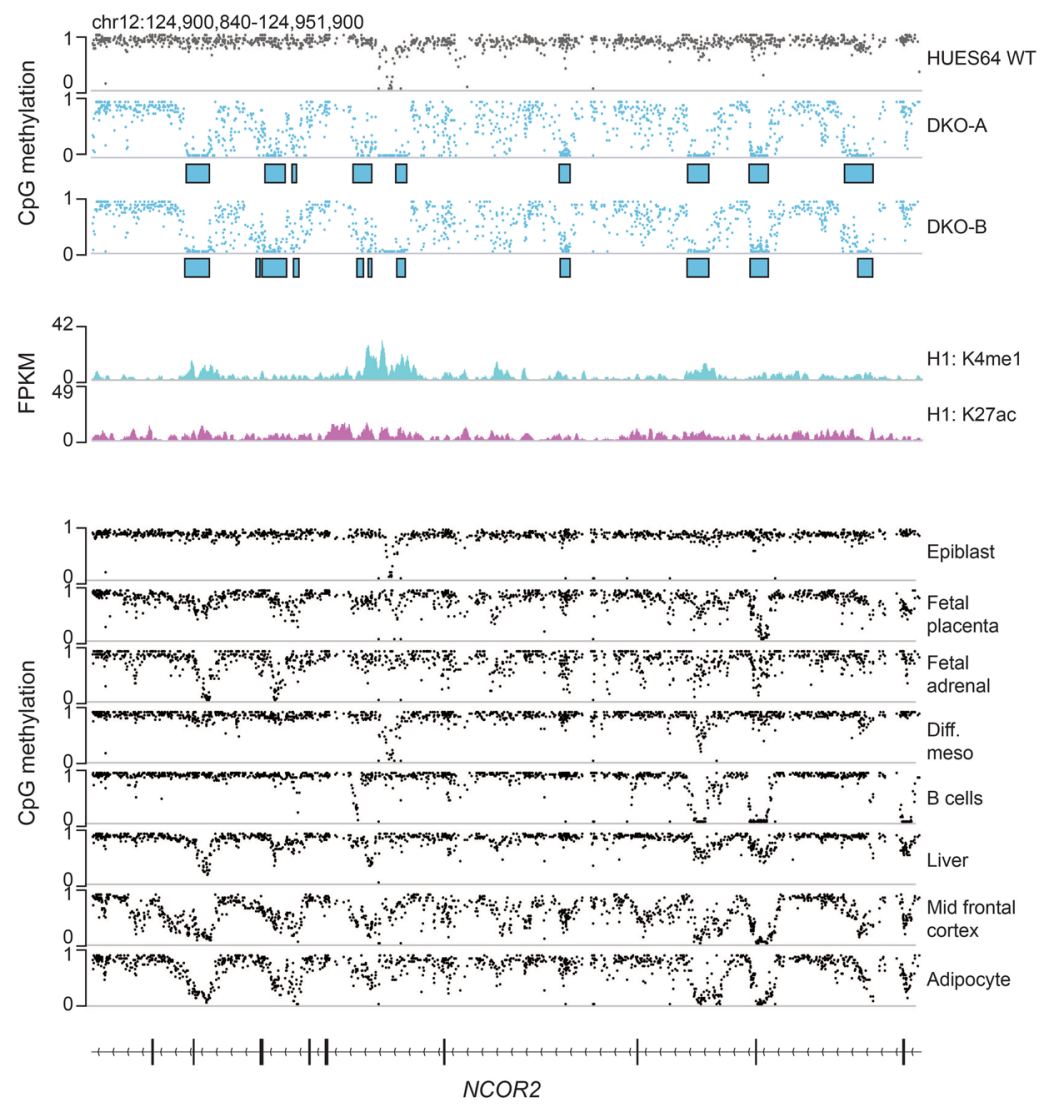

f

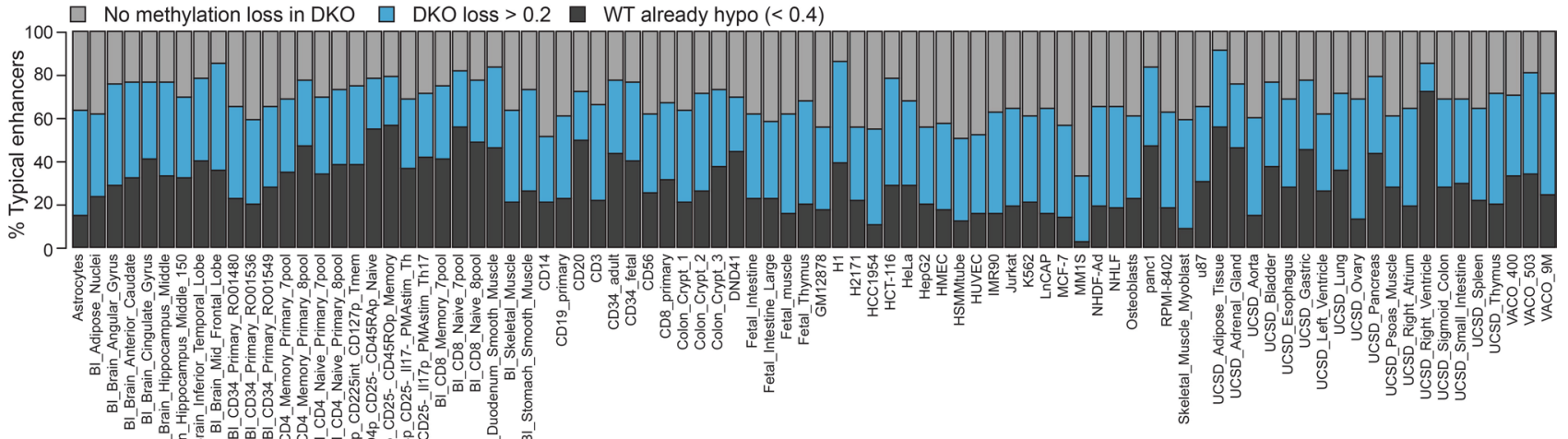

g

$\mathrm{h}$
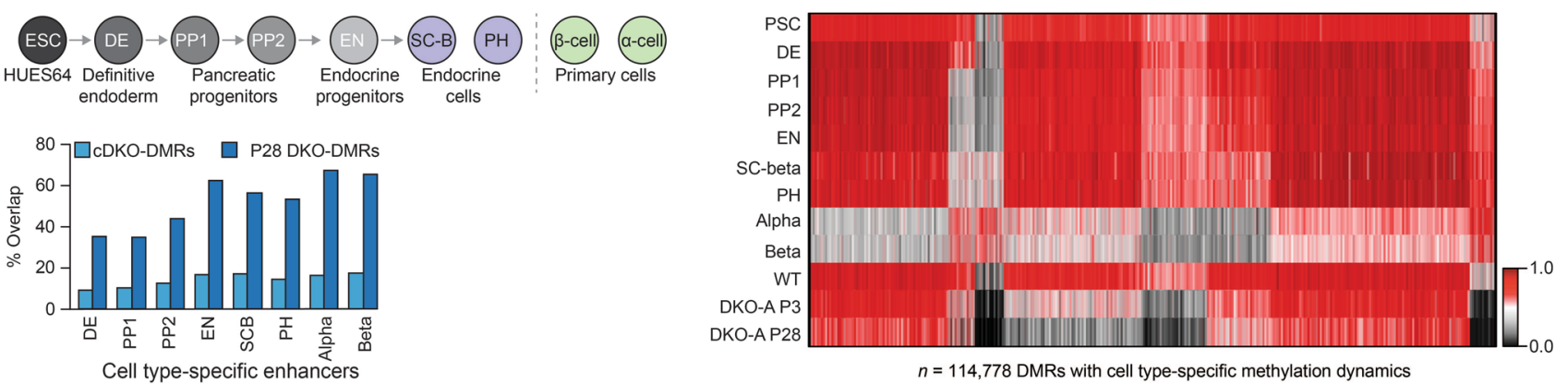

Extended Data Fig. 4 | See next page for caption. 
Extended Data Fig. 4 | cDKO-DMRs overlap with tissue-specific enhancers. a, TET1 enrichment (from ref. ${ }^{21}$ ) for LINE 5'UTRs and active H1 enhancers. b. A region within the gene NCOR2 that has many CDKO-DMRs. These lose methylation in different somatic tissues as shown below. H3K27ac and $\mathrm{H} 3 \mathrm{~K} 4 \mathrm{me} 1 \mathrm{tracks}$ display ENCODE data derived from H1 ESCs. Methylation tracks are from ref. ${ }^{6}$. c, The percentage of overlap with putative somatic enhancers (defined in ref. ${ }^{32}$ ) for class 1 and class 2 cDKO-DMRs, an equal number of same-sized randomly selected regions, H3K4me1 peaks in ESCs $(\mathrm{H} 1)$ and $1 \mathrm{~kb}$ tiles with matched $\mathrm{CpG}$ density to cDKO-DMRs (3.1-3.3\%). d, B cell enhancers (defined in ref. ${ }^{32}$ ) separated by methylation levels in WT ESCs, DKO ESCs and B cells. e, Composites showing methylation levels in passage (P) 3 and 28 DKO ESCs for P3 and P28 DKO-DMRs. f, For 86 different previously defined putative enhancer sets ${ }^{32}$, the stacked bar plots display the proportion that are already hypomethylated in WT ESCs, lose methylation in DKO cells (WT - DKO difference > 0.2) or remain methylated in DKO cells. For this analysis, P28 DKO was used. $\mathbf{g}$, Schematic of in vitro pancreatic islet cell differentiation (from ref. ${ }^{33}$ ). $\mathbf{h}$, The proportion of cDKO-DMRs or P28 DKO-DMRs that overlap with each set of cell type specific enhancers. Enhancers were previously defined ${ }^{33}$. i, For regions defined as showing dynamic methylation changes during differentiation to beta islet cells ${ }^{33}$, methylation levels are shown for these cell types as well as HUES64 WT and DKO-A ESCs. 
a

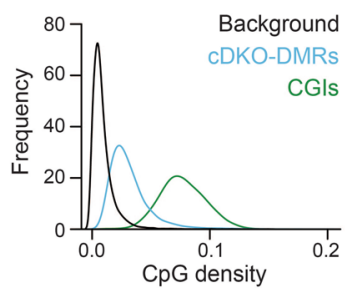

e

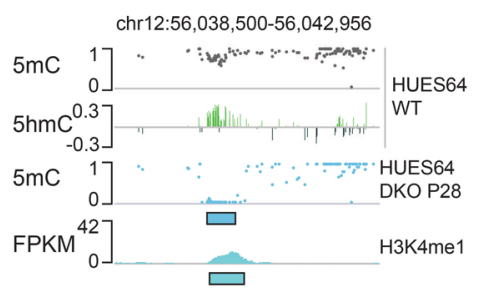

b

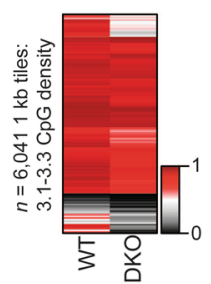

f

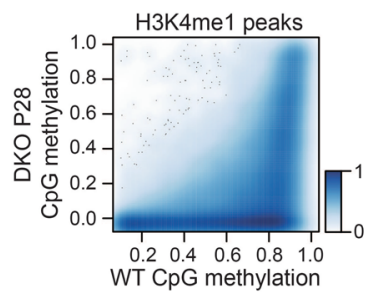

d

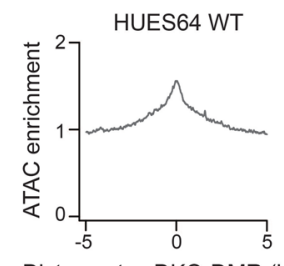

Distance to cDKO-DMR (kb)

\section{g}

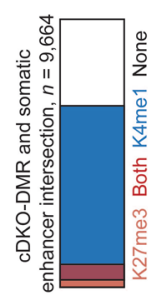

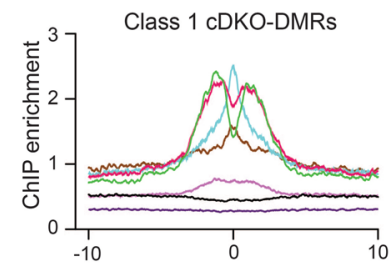

H3K27ac H3K27me3 H3K36me3 H3K9me3 H3K4me1 H3K4me2 H3K4me3

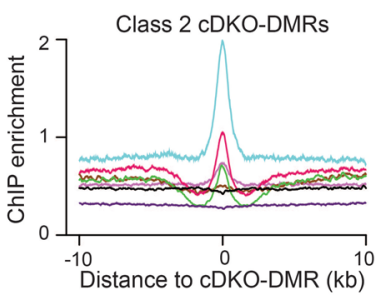

h

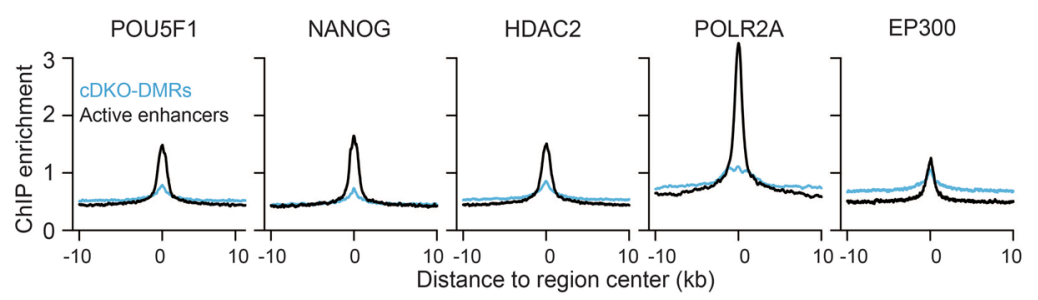

i

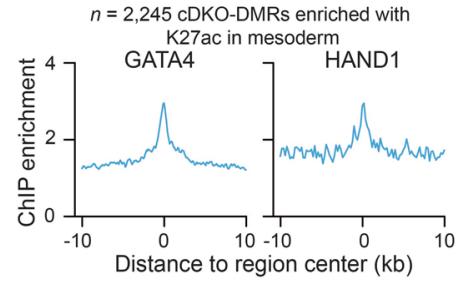

j

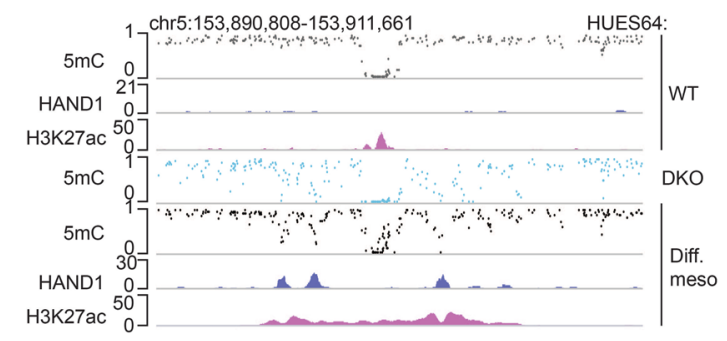

I

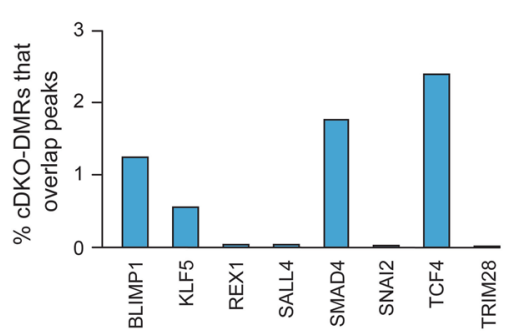

m

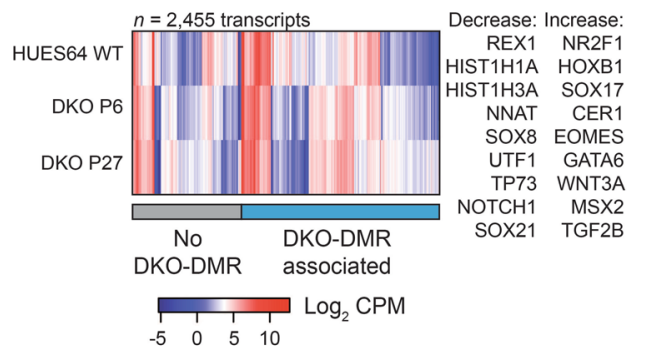

k
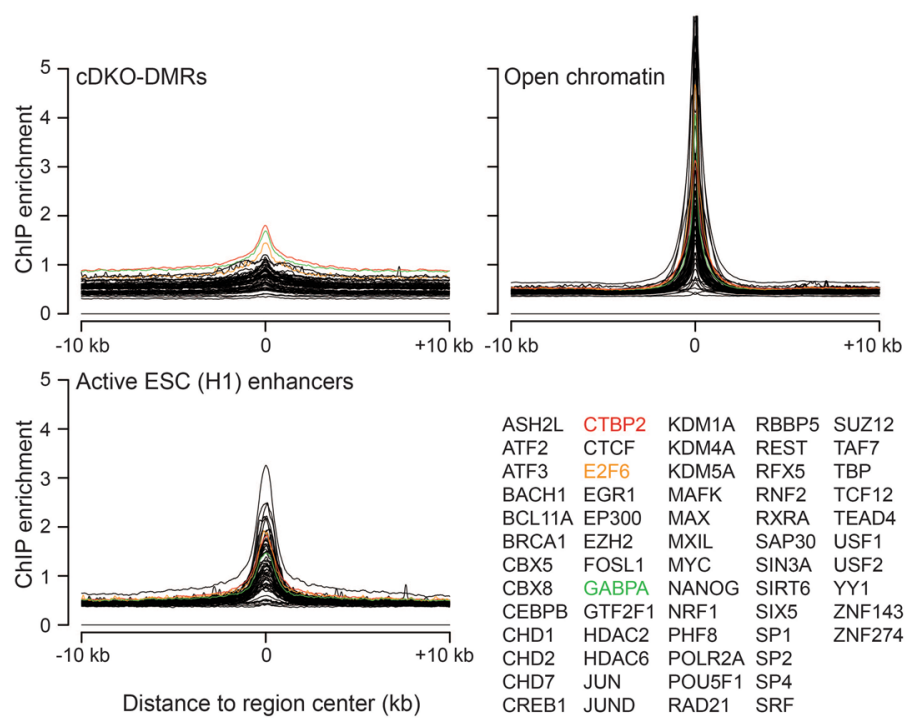

ASH2L CTBP2 KDM1A RBBP5 SUZ12 ATF2 CTCF KDM4A REST TAF7 ATF3 E2F6 KDM5A RFX5 TBP BACH1 EGR1 MAFK RNF2 TCF12 BCL11A EP300 MAX RXRA TEAD4 BRCA1 EZH2 MXIL SAP30 USF1 CBX5 FOSL1 MYC SIN3A USF2 CBX8 GABPA NANOG SIRT6 YY1 CEBPB GTF2F1 NRF1 SIX5 ZNF143 CHD1 HDAC2 PHF8 SP1 ZNF274 CHD2 HDAC6 POLR2A SP2 CHD7 JUN POU5F1 SP4 CREB1 JUND RAD21 SRF

$\mathrm{n}$

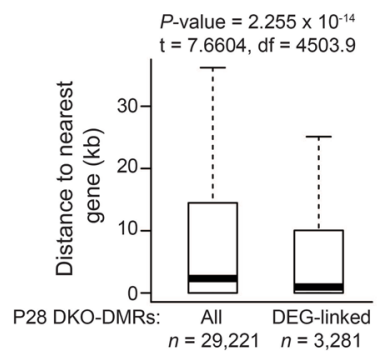

Extended Data Fig. 5 | See next page for caption. 
Extended Data Fig. 5 | Chromatin states and transcription factor interaction at cDKO-DMRs. a, CpG density for randomly selected background regions, cDKO-DMRs and CGIs. b, For $1 \mathrm{~kb}$ tiles with CpG density matched to cDKO-DMRs (3.1-3.3\%), the mean methylation in HUES64 WT and DKO-A is shown. Methylation loss is not dependent on CpG density. c, ATAC-seq signal enrichment (fragments per bp, per peak) for cDKO-DMRs. d, Enrichment for chromatin modifications (ENCODE, H1 ESCs; fragments per bp, per peak) as distance from cDKO-DMRs (fragments per bp, per peak). e, A representative locus showing enrichment of $\mathrm{H} 3 \mathrm{~K} 4 \mathrm{me} 1$ and $5 \mathrm{hmC}$ at a cDKO-DMR. $\mathrm{P}=$ passage. $\mathbf{f}$, Methylation levels for ENCODE-defined H3K4me1 peaks ( $\geq 3 \mathrm{CpGs,}$ methylation $\geq 0.1$ in WT ESCs, $n=71,665)$ for WT and DKO cells at P28. Intensity of blue shading indicates the relative density of data points. Many regions remain methylated indicating that $\mathrm{H} 3 \mathrm{~K} 4 \mathrm{me} 1$ enrichment does not predict demethylation. $\mathbf{g}$, For cDKO-DMRs that overlap with putative somatic enhancers, the proportion that are $\mathrm{H} 3 \mathrm{~K} 4 \mathrm{me} 1$ and/or H3K27me3 enriched is displayed. Overlap with both marks indicates a poised chromatin state that is more common for already hypomethylated poised enhancers. h, ChIP-seq enrichment (ENCODE, H1 ESCs; fragments per bp, per peak) for different enhancer-associated factors at cDKO-DMRs and active ESC enhancers. i, HAND1 (GSM1505812) and GATA4 (GSM1505644) enrichment in HUES64 differentiated mesoderm ( $r$ ef. ${ }^{34}$ ) for cDKO-DMRs that show H3K27ac (GSM1505669) enrichment in mesoderm (indicating an active enhancer-like chromatin state). $\mathbf{j}$, Browser tracks displaying regions that lose methylation while gaining H3K27ac and transcription factor (HAND1) binding upon differentiation to mesoderm. The same CpGs also lose methylation in DKO ESCs. ChIP-seq data is from ref. ${ }^{34}$. $\mathbf{k}$, Enrichment for TF binding (ENCODE, H1 ESCs) at cDKO-DMRs, open DNA (based on HUES64 ATAC-seq) and active ESC enhancers is displayed. The most enriched factors at cDKO-DMRs (CTBP2, E2F6 and GABPA) show only minimal enrichment that could be confounded by the more open chromatin at cDKO-DMRs. No factor showed unique enrichment that would support their role as candidate factors for TET/DNMT recruitment. I, The percentage of cDKO-DMRs that overlap with different TF binding sites, defined by ChIP-seq peak data (ref. ${ }^{34}$ ). Overlap is consistently low. $\mathbf{m}$, Expression levels for differentially expressed genes between WT and DKO with examples to the right. P = passage. Each P28 DKO-DMR was assigned to its nearest gene, and those with a DKO-DMR assigned are highlighted with the blue bar. $\mathbf{n}$, The distribution of absolute distance to the nearest gene for P28 DKO-DMRs. 'DEG-linked' refers to DKO-DMRs that have been assigned to a differentially expressed gene by proximity. Statistics refer to a 2-sided T-test. For the box plots: median is shown in bold, box displays interquartile range, whiskers extend to $1.5 x$ the interquartile range. 
a

DNMT3A: top strand $=$ coding, $630-908 a a=$ catalytic domain $\mathrm{mm} 10$, chr12:3,895,552-3,895,590, exon 7

$$
A \stackrel{215}{K} \vee V \quad I \quad A \quad V \quad \stackrel{220}{M} \text { N A } V \text { E } \stackrel{225}{E}
$$

WT + GCCAAGGTAATGCAGTAATGAATGCTGTG AAGAG

$$
\begin{gathered}
215 \\
\text { A }
\end{gathered}
$$

DKO + GCCAAGGTAATTGCAGT------_GCTGTGGAAGAGA--90bp--CCACCCCTGAGCCAGTAG

Allele 1 - CGGTTCCATTAACGTCA------CGACACCITCTCT--90bp--GGTGGGGACTCGGICATC

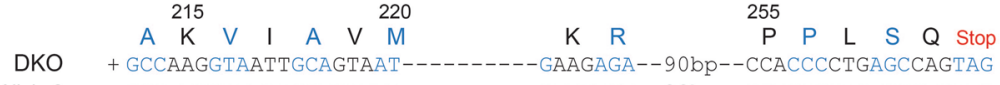
Allele 2 - CGGTTCCATTAACGTCATTA--------CTTCTCT--90bp--GGTGGGGACTCGGTCATC

mm10, chr12:3,902,697-3,902,760, exon 19

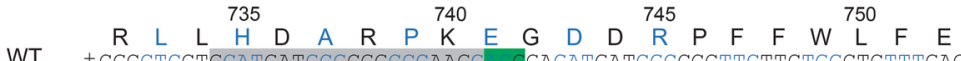

$$
\begin{aligned}
& \text { WT +CGCCTCCTGCATGATGCGCGGCCCAAG GGAGATGATCGCCCCTTCTTCTGGCTCTTTGA } \\
& \text { - GCGGAGGACGTACTACGCGCCGGGTTCCTCCCTCTACTAGCGGGGAAGAAGACCGAGAAACT } \\
& \text { R L L } \stackrel{735}{H} \text { D } \\
& \text { DKO + CGCCTCCTGCATGATG } \\
& \text { A P } \quad \stackrel{740}{L} \text { L A L Stop } \\
& \text { Allele } 1 \text { - GCGGAGGACGTA-- }
\end{aligned}
$$

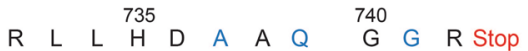

DNMT3B: top strand $=$ coding, $581-859$ aa $=$ catalytic domain

$\mathrm{mm} 10$, chr2:153,661,278-153,661,553, exon 3

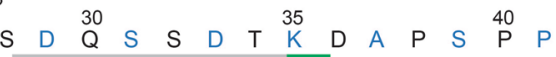

WT + TGGGATCATG--204bp--AGTGACCAGTCCTCAGACACGA GATGCTCCCTCACCCCCA

$$
\begin{array}{llllllll}
35 & & & & & 40 & & \\
K & D & A & P & S & P & P
\end{array}
$$
Allele $1-$ ACCCTAG----204bp-n-n

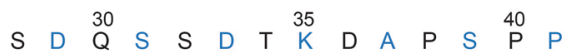

DKO + TGGGATCATG--204bp--AGTGACCAGTCCTCAGACACGAAGGATGCTCCCTCACCCCCA Allele 2 - ACCCTAGTAC--204bp--TCACTGGTCAGGAGTCTGTGCTTCCTACGAGGGAGTGGGGGT

mm10, chr2:153,677,537-153,677,632, exon 20

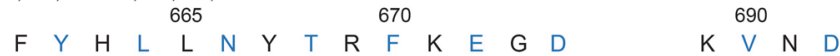

$$
\begin{aligned}
& \text { + TTTTACCACTTECTGAATTATACCCGCCCCAAG AGGGCGACGGG }
\end{aligned}
$$

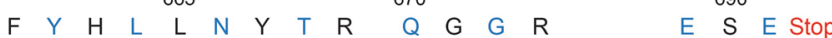
Allele 1+2 - AAAATGGTGAACGACTTAATATGGGCGGGGTTCCTCCCGCT--42bp--CITTCACTTACTG

d

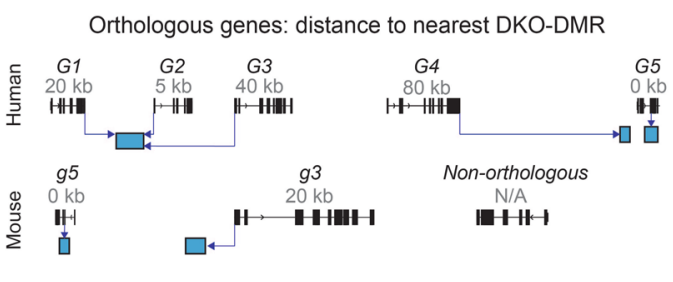

$n=3,888$ mouse EpiSC DKO-DMRs, $n=11,430$ human ESC cDKO-DMRs

Extended Data Fig. 6 | See next page for caption. b

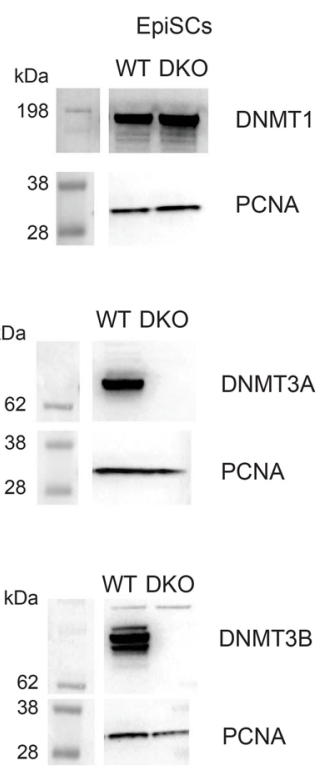

C
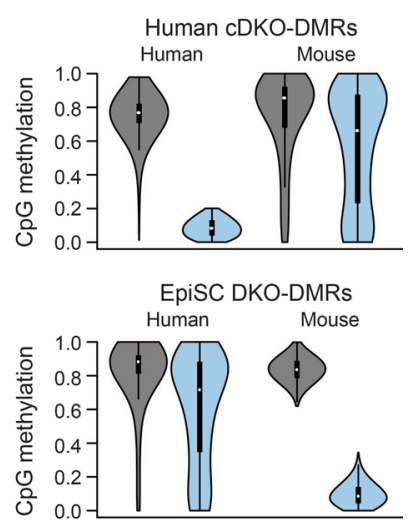

$\square$ WT $\square$ DKO

e

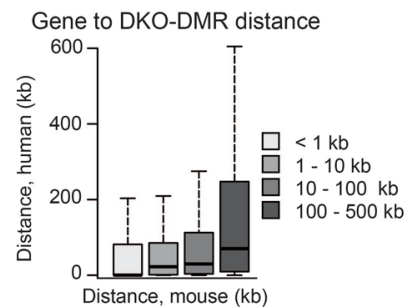

Gene body DKO-DMRs

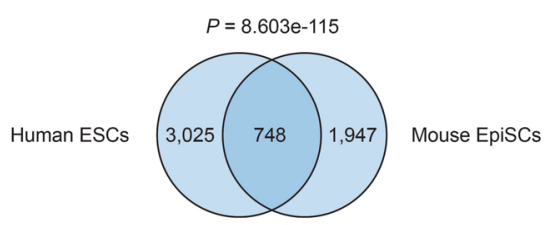

All orthologous genes: $n=16,727$ 
Extended Data Fig. 6 | Generation of DNMT3A and 3B knockout mouse EpiSCs. a, Sequencing data showing the Dnmt3a and Dnmt3b mutations introduced into mouse DKO EpiSCs. Two different sgRNAs were used for each gene. Green box = PAM sequence, grey box = sgRNA sequence. Codons are colored black or blue, grey text denotes non-coding DNA. The respective single letter amino acid (aa) and protein sequence number is displayed above the DNA. b, Western blots showing protein levels for DNMT1, DNMT3A and DNMT3B in WT and DKO EpiSCs. PCNA is used as a loading control. Full blots in Source Data Fig. 2. Western blots were performed twice with consistent results. c, Violin plots display methylation levels for cDKO-DMRs and EpiSC DKO-DMRs in WT and DKO cells for each species. The UCSC LiftOver tool was used to map DKO-DMRs between species. Violin plots extend from the data minima to the maxima with the white dot indicating median, thick bar showing the interquartile range and thin bar showing $1.5 x$ interquartile range. d, Schematic depicting how orthologous genes shared between human and mouse were assigned to the nearest DKO-DMR (left). Each gene was given a number (in kb) denoting the distance to the nearest DKO-DMR either up- or down-stream. Box plots for genes that were binned based in their distance to the nearest DKO-DMR in EpiSCs (shown in legend key), displaying the distance to the nearest DKO-DMR in human ESCs (right). A matched trend between distances in mouse and human cells in evident. For the box plots: the median is shown in bold, the box displays interquartile range and whiskers extend to 1.5 times the interquartile range. e, Venn diagram showing the overlap of orthologous genes that have a DKO-DMR within the gene body ( 0 kb away, as shown in panel d) in mouse EpiSCs and human ESCs. This overlap was highly significant (hypergeometric test). 
a

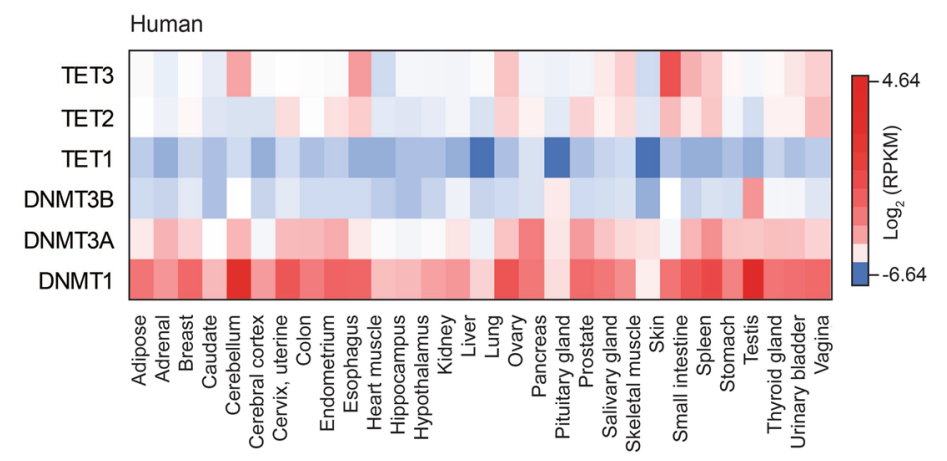

$\mathrm{b}$

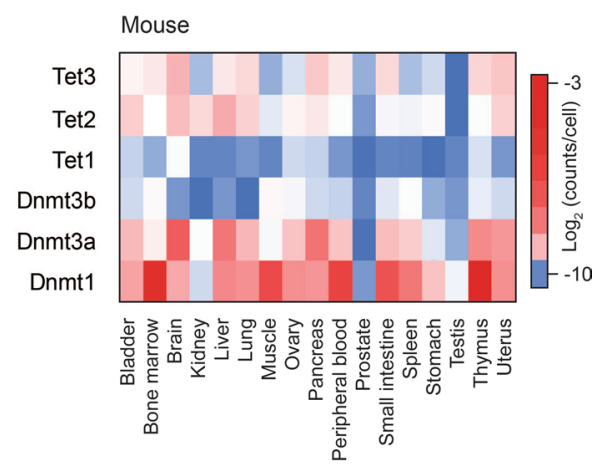

Extended Data Fig. 7 | DNMT and TET expression in somatic tissues. a, Heat map showing Log 2 RPKM for DNMTs and TETs across human somatic tissues, based on data from the human protein atlas (ebi.ac.uk). b. Heat map showing Log $_{2}$ transcript counts per cell for murine somatic tissues, taken from single cell mouse atlas data. 
a

Mouse ESCs

Dnmt3a: top strand = coding, 630-908aa = catalytic domain

$\mathrm{mm} 10$, chr12:3,902,697-3,902,740, exon 19

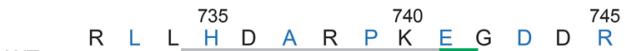

$$
\begin{aligned}
& \text { WT + CGCCTCCTGCATGATGCGCGGCCCAAGG GGAGATGATCGC }
\end{aligned}
$$

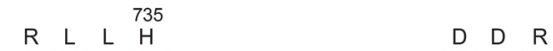

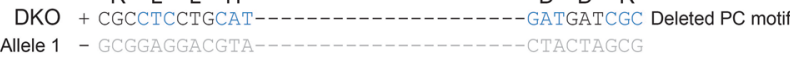

$$
\text { R L L } \stackrel{735}{H} \text { D A }
$$

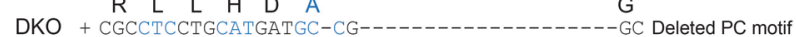

Allele 2 - GCGGAGGACGTACTACG-GCCGGGTTCCTCCCTCTACTAGCd

Dnmt3b: top strand $=$ coding, $581-859$ aa $=$ catalytic domain mm10, chr2: 153,677,540-153,677,632, exon 20

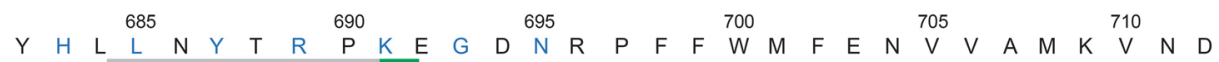

WT + TACCACTTGCTGAATTATACCCGCC-CCA GAGGGCGACAACCGTCCATTCTTCTGGATGTTCGAGAATGTTGTGGCCATGAAAGTGAATGAC

- ATGGTGACGACTTAATATGGGGG-GGTCCTCCCGCTGTGGCAGGAAGAAGACCTACAAGCTCTTACAACACCGGTACTTTCACTTACTG

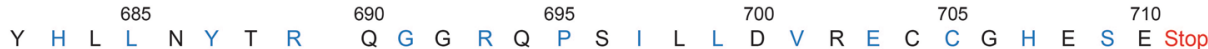

DKO + TACCACTTGCTGAATTATACCCGCC---AAGGAGGGCGACAACCGTCCATTCTTCTGGATGTTCGAGAATGTTGTGGCCATGAAAGTGAATGAC

Allele 1 - ATGGTGAACGCTTAATATGGGCGG---TTCCTCCCGCTGTTGGCAGGTAAGAAGACCTACAAGCTCTTACAACACCGGTACTTTCACTTACTG

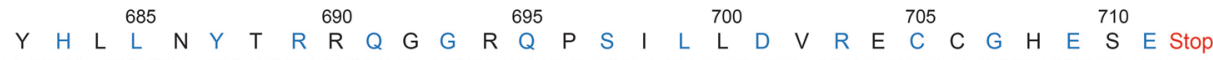

DKO + TACCACTTGCTGAATTATACCCGCCGCCAAGGAGGGCGACAACCGTCCATTCTTCTGGATGTTCGAGAATGTTGTGGCCATGAAAGTGAATGAC Allele 2 - ATGGTGAACGACTTAATATGGGCGGCGGTTCCTCCCGCTGTTGGCAGGTAGAAGACCTACAAGCTCTTACAACACCGGTACTTTCACTTACTC

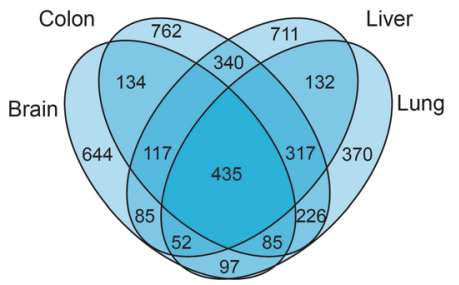

d

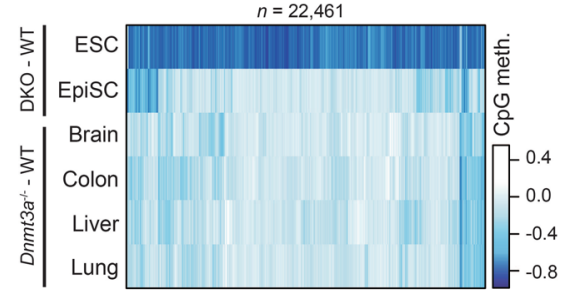

f

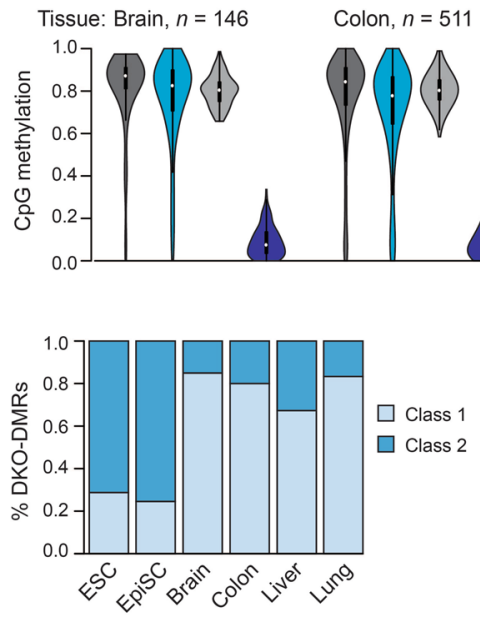

j

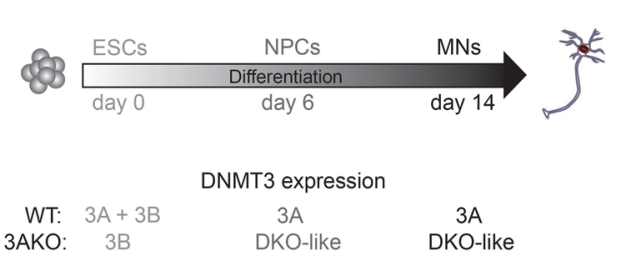

3AKO: $3 B \quad$ DKO-like $\quad 3$ DKO-like

i

k

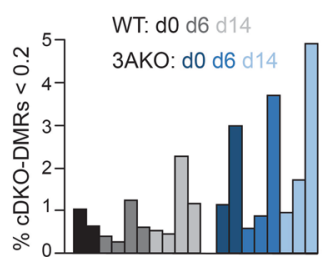

e

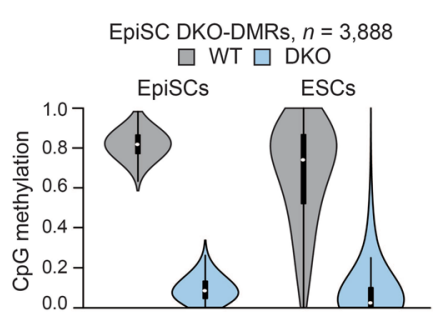

g

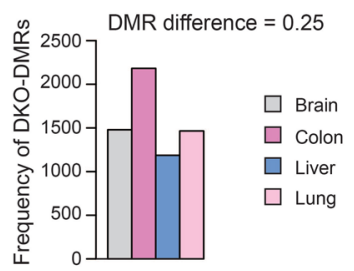

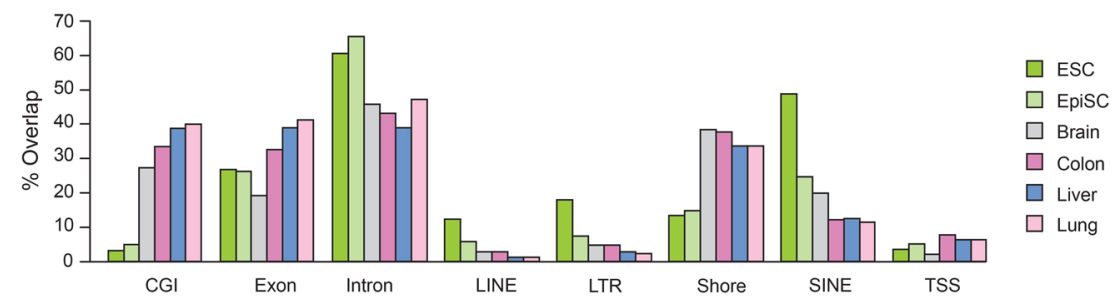

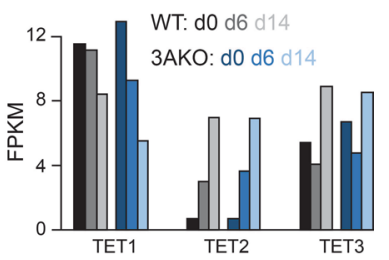

Extended Data Fig. 8 | See next page for caption. 
Extended Data Fig. 8 | DKO-DMRs are associated with pluripotency. a, Sequence mutations introduced into the DKO ESCs. Green box = PAM sequence, grey box $=$ sgRNA. Codons are colored black or blue, grey text denotes non-coding DNA. Amino acid (aa) and protein sequence number are displayed above the DNA. b, Western blots for WT, DNMT3A ${ }^{-/-}(3 A K O)$, DNMT3B ${ }^{-/-}$(3BKO) and double knockout (DKO) mouse ESCs. GAPDH or H3 were used as loading controls. Full blots in Source Data Fig. 3. Western blots were performed three times with consistent results. c, Overlap between DKO-DMRs called in each Dnmt3a-/- tissue. d, The difference in methylation between WT and Dnmt3a-/- tissues, ESCs or EpiSCs. (e) Methylation levels for EpiSC DKO-DMRs in EpiSCs and ESCs. Violin plots extend from the data minima to the maxima, white dot indicates median, thick bar shows the interquartile range and thin bar shows 1.5x interquartile range. f, Methylation levels for tissue-specific DKO-DMRs in WT and Dnmt3a-/- E6.5 epiblast and 8 day old tissues (Dnmt3a-/- E6.5 epiblast data from GSE137337). Somatic DKO-DMRs were fully methylated in Dnmt3a ${ }^{-/-}$embryos following implantation due to DNMT3B expression, hence must have lost methylation at a later stage. Violin plots extend from the data minima to the maxima, white dot indicates median, thick bar shows the interquartile range and thin bar shows $1.5 \mathrm{x}$ interquartile range. $\mathbf{g}$, The number of DKO-DMRs identified when using a reduced stringency of 0.25 differential methylation instead of 0.6 to compensate for mixed cell types. We identified more DKO-DMRs $(1,186-2,182)$ but still many less than in ESCs, and this may include more false positives. $\mathbf{h}$, The percentage of DKO-DMRs that fall into class 1 or 2 (described in Figure 1).

i, The overlap of DKO-DMRs with genomic features. Categories are not exclusive. CGI = CpG island, TSS = transcription start site. $\mathbf{j}$, Schematic showing the differentiation of human ESCs to motor neurons (MNs) via neuronal progenitor cells (NPCs). For WT and 3AKO ESCs expression of DNMT3A and DNMT3B is displayed across the time course. After day two, 3AKO cells are 'DKO-like' as they do not express either DNMT3. $\mathbf{k}$, The percentage of cDKO-DMRs with mean methylation $<0.2$ in each sample during differentiation. Only $1.3 \%$ and $2.4 \%$ of cDKO-DMRs lost methylation for WT and $3 A K O$ MNs respectively. I, Expression of TET1-3 in WT and 3AKO ESCs during differentiation. WGBS and RNA-seq data for $\mathbf{k}$ and $\mathbf{i}$ are from ref. ${ }^{39}$. 
a

27,363,796 matched CpGs:

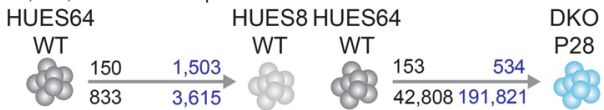

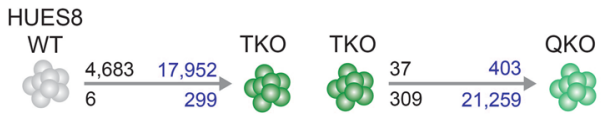

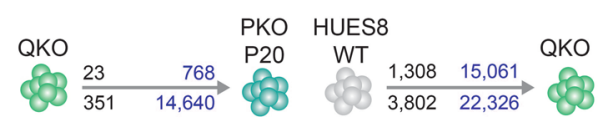

HUES8 PKO PKO

\begin{tabular}{lllll} 
WT & & P20 TKO & P20 \\
\hline & & &
\end{tabular}

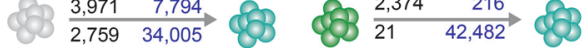

\begin{tabular}{|c|c|c|}
\hline$R_{R}$ & Incr. & $\begin{array}{l}8 / 10 \text { CpGs, diff }>0.6, p<0.01 \\
5 / 8 \mathrm{CpGs} \text {, diff }>0.25, p<0.01\end{array}$ \\
\hline 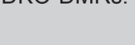 & Decr. & $\begin{array}{r}\text { Total: } 52,686 \\
238,497\end{array}$ \\
\hline
\end{tabular}

d
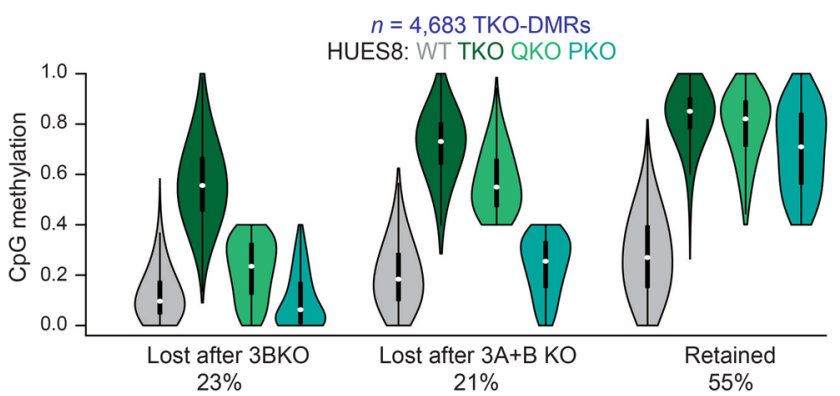

b

C
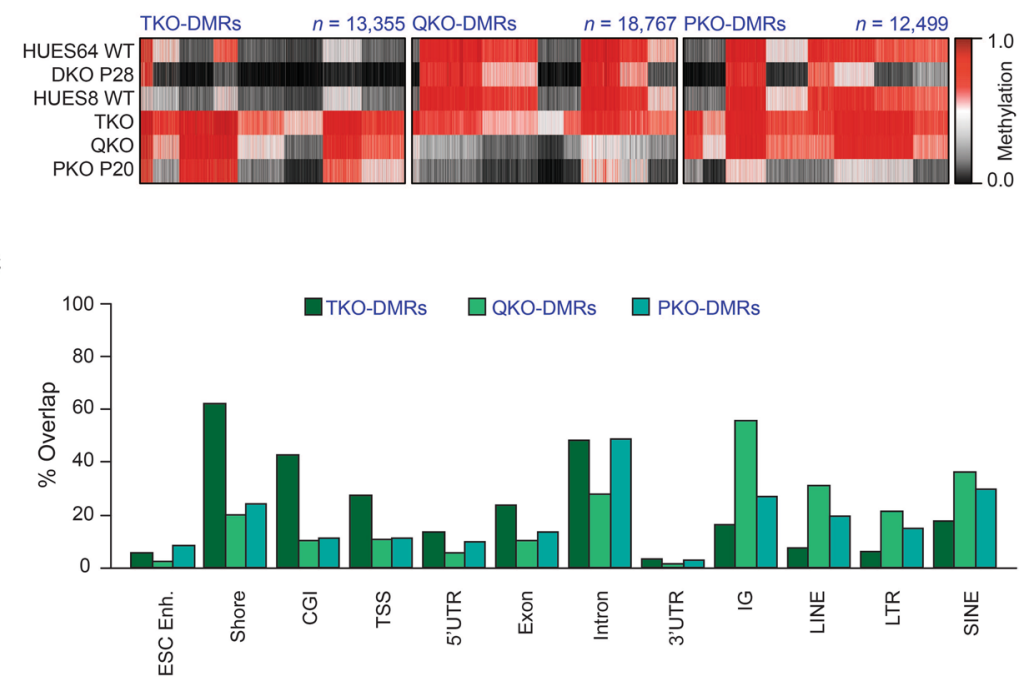

e

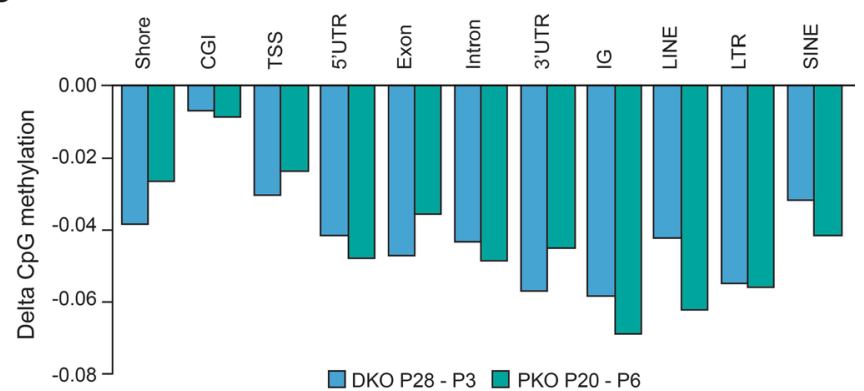

f

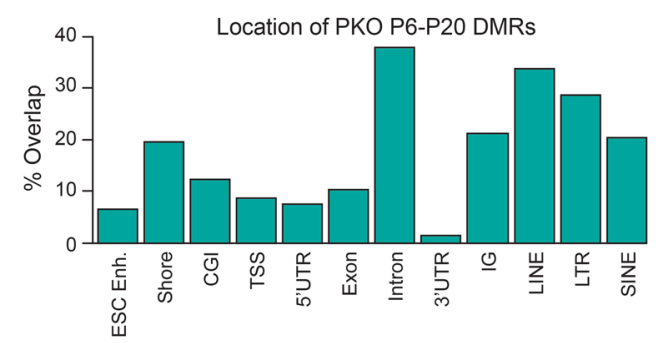

g

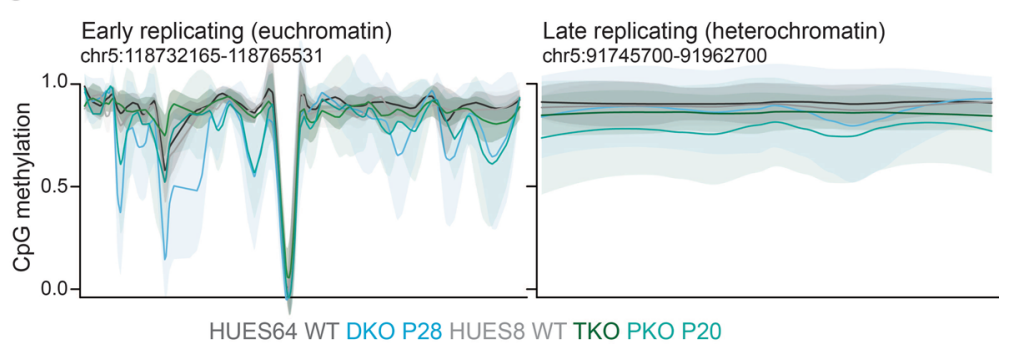

Extended Data Fig. 9 | Identification of DMRs across human ESCs. a, Schematic illustrating how DMR calling was performed between each pair of samples. The number of DMRs that gain methylation (Incr.) in the cell type shown to the right is located above the arrow, and the number of DMRs that lose methylation (Decr.) is shown below the arrow. As we identified only few regions for other comparisons that met the same criteria as for DKO-DMRs (stringent DMRs; black) we also used less stringent criteria $(\Delta>0.25)$ to define DMRs (blue). P = passage. b, CpG methylation of TKO-, QKO- and PKO-DMRs detected with lower stringency as defined in panel $a$. The $\mathrm{n}$ is lower than shown in panel a due to drop-out from requiring $10 \mathrm{x}$ coverage per region. $\mathbf{c}$, Location of the DMRs shown in b. Features are not exclusive. Enh. $=$ enhancer, $C G I=C p G$ island, TSS $=$ transcription start site, IG $=$ intergenic. As expected and previously reported in ref. ${ }^{21}$, the majority of DMRs that occur in the absence of TETs were CGI promoters or shores of developmental genes that may be more susceptible to hypermethylation due to lack of transcriptional activity. $\mathbf{d}$, Low stringency TKO-DMRs are grouped by their methylation status in QKO and PKO cells. Of the TKO-DMRs, 23\% lost the aberrantly gained methylation upon KO of DNMT3B (in QKO), 21\% only upon further loss of DNMT3A (PKO) and 56\% remained highly methylated despite loss of both DNMT3s, pointing to the importance of TET activity in maintaining these regions in a hypomethylated. e, The difference in methylation over passaging across each genomic feature. $\mathbf{f}$, The location of DMRs identified between PKO at P6 and P20. Categories are not exclusive. $\mathbf{g}$, Loess-smoothed regression for methylation levels across a representative early-replicating region, where methylation in DKO cells decreases the most, (left) and a representative late-replicating region, where PKO cells show the strongest methylation loss (right). 
a

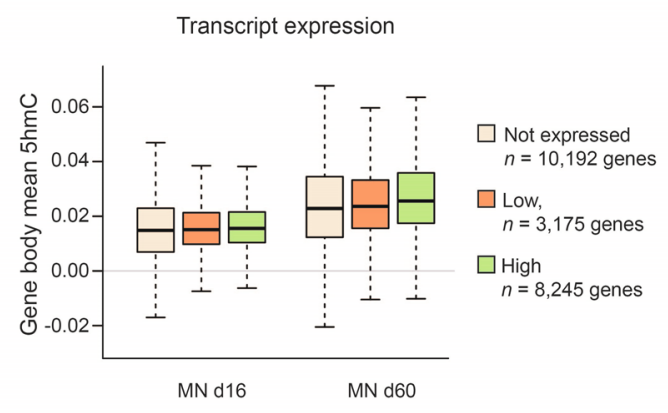

b

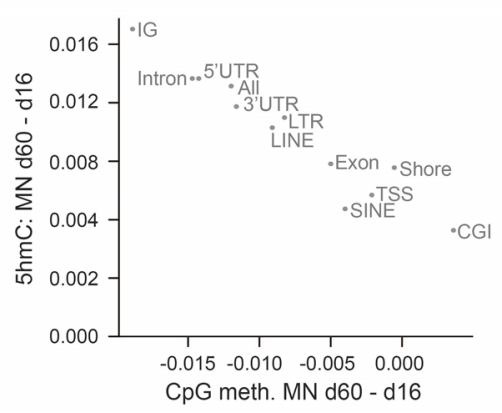

C

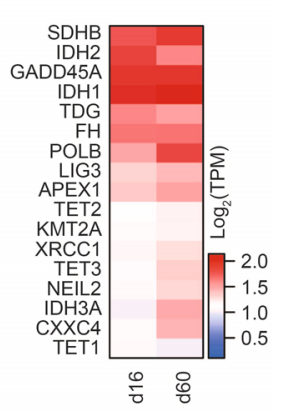

d

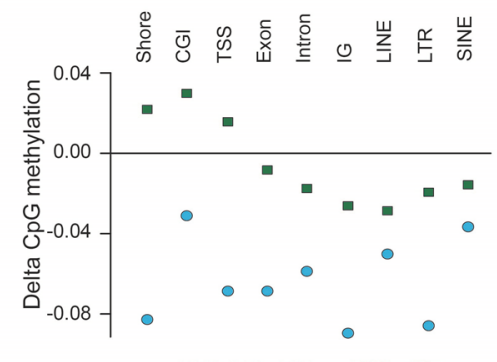

DKO P28 - WT TKO - WT

f

Methylated

locus, WT ESCs: mC-turnover

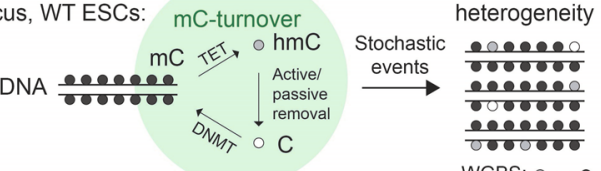

h

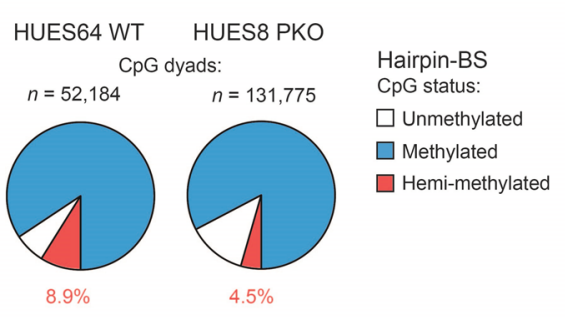

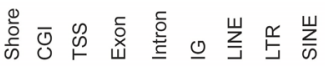

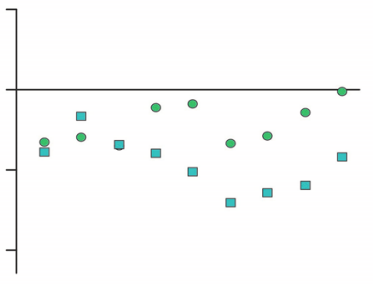

QKO - TKO PKO P20-QKO

\section{e}

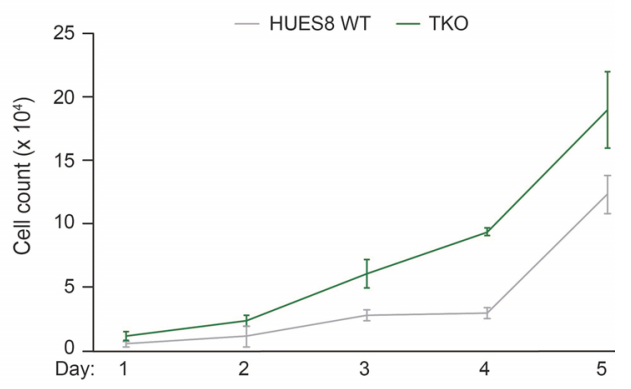

g

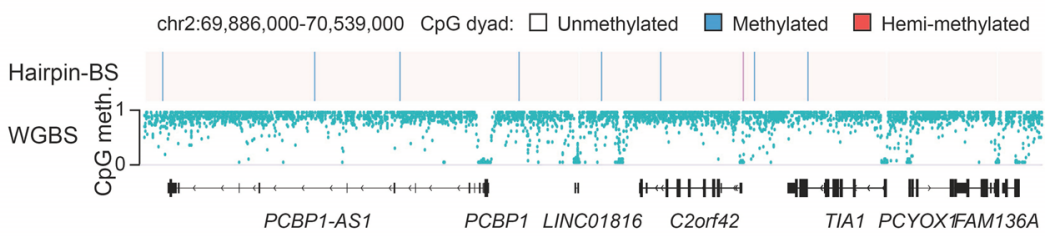

i

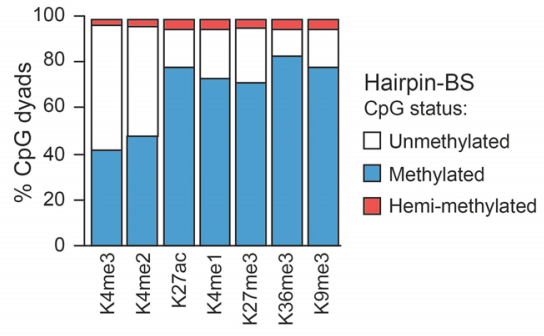

k
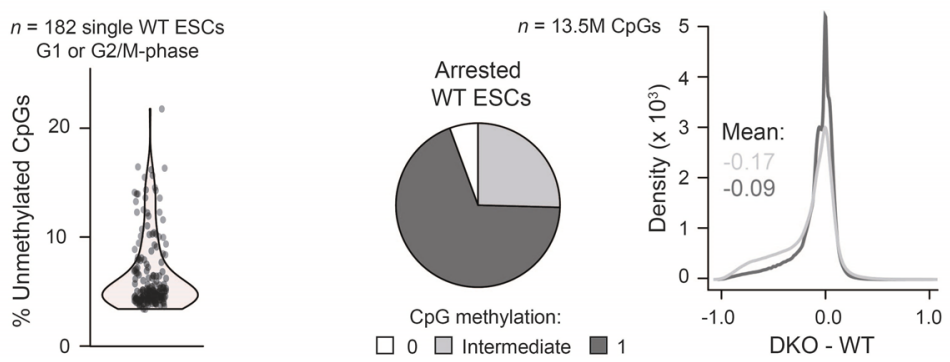

Extended Data Fig. 10 | See next page for caption. 
Extended Data Fig. 10 | Broad TET activity results in genome-wide 5hmC enrichment and methylation heterogeneity. a, Mean 5hmC level in HUES64 differentiated motor neurons ( $M N$ ) over gene bodies grouped into categories according to their expression status, $d=$ day. The median is shown in bold, the box displays interquartile range and whiskers extend to $1.5 \mathrm{x}$ the interquartile range. $\mathbf{b}$, The difference ( $\mathrm{d} 60 \mathrm{-d}$ 16) in $5 \mathrm{hmC}$ vs. methylation for MNs at different genomic features. CGI = CpG island, TSS = transcription start site, IG = intergenic. c, Transcript expression for genes involved in TET-mediated demethylation and base excision repair pathways. $\mathbf{d}$, For genomic features, the mean difference in methylation between ESCs is displayed. e, Cell counts for HUES8 WT and TKO cells over $5 \mathrm{~d}$ culture. Center points show the mean and error bars display the standard deviation for three independent cell culture replicates. $\mathbf{f}$, Schematic showing how stochastic turnover of cytosine modification can lead to intercellular heterogeneity. $\mathrm{mC}=$ methylcytosine (black circle), $5 \mathrm{hmC}=$ hydroxymethylcytosine (grey circle), $C=$ unmethylated cytosine (white circle). $\mathbf{g}$, Representative browser tracks showing the methylation status for $\mathrm{CpG}$ s detected using hairpin bisulfite sequencing (Hairpin-BS; top) and WGBS (bottom). Each CpG covered by hairpin-BS is colored according to dyad methylation state. $\mathbf{h}$, Proportion of CpG dyads that were methylated, unmethylated or hemi-methylated in arrested HUES64 WT or PKO ESCs. i, For regions enriched with different chromatin marks (ENCODE, $\mathrm{H} 1 \mathrm{ESCs}$ ), the proportion of $\mathrm{CpG}$ dyads with each methylation status is displayed. $\mathbf{j}$, The percentage of $\mathrm{CpG}$ dyads that were hemi-methylated according to location within each genomic feature. TE = active typical enhancer. $\mathbf{k}$, Single cell RRBS data showing the percentage of unmethylated CpGs per cell. The violin plot extends from the data minima to the maxima where each dot is a single cell. Of CpGs that are normally methylated in bulk WGBS ( $\geq 0.9$ ), we observed that $6.6 \% \pm 3.37$ showed zero methylation in individual cells. These may have been targeted by TETs and are likely an underestimate as $5 \mathrm{hmC}$ cannot be discriminated from $5 \mathrm{mC}$ with our RRBS based approach. I, The proportion of CpGs with zero, intermediate (not exactly zero or one) or one methylation status for arrested WT ESCs. In bulk populations, ESC arrest removes replication-associated heterogeneity and increases the proportion of $\mathrm{CpGs}$ that are reported as fully methylated. However, $\sim 25 \%$ remain intermediate. The density plot shows methylation loss for intermediate and fully methylated $\mathrm{CpGs}$. Intermediate $\mathrm{CpGs}$ show a greater loss, suggesting they may be more frequently targeted by TETs. Hence TET activity may contribute to intermediate methylation levels in bulk populations. 


\section{Reporting Summary}

Nature Research wishes to improve the reproducibility of the work that we publish. This form provides structure for consistency and transparency in reporting. For further information on Nature Research policies, see Authors \& Referees and the Editorial Policy Checklist.

\section{Statistics}

For all statistical analyses, confirm that the following items are present in the figure legend, table legend, main text, or Methods section.

$\mathrm{n} / \mathrm{a}$ Confirmed

\ The exact sample size $(n)$ for each experimental group/condition, given as a discrete number and unit of measurement

Х

A statement on whether measurements were taken from distinct samples or whether the same sample was measured repeatedly

$\varnothing$ The statistical test(s) used AND whether they are one- or two-sided

Only common tests should be described solely by name; describe more complex techniques in the Methods section.

$\searrow$ A description of all covariates tested

\ $\square$ A description of any assumptions or corrections, such as tests of normality and adjustment for multiple comparisons

A full description of the statistical parameters including central tendency (e.g. means) or other basic estimates (e.g. regression coefficient)

AND variation (e.g. standard deviation) or associated estimates of uncertainty (e.g. confidence intervals)

For null hypothesis testing, the test statistic (e.g. $F, t, r$ ) with confidence intervals, effect sizes, degrees of freedom and $P$ value noted Give $P$ values as exact values whenever suitable.

Х $\square$ For Bayesian analysis, information on the choice of priors and Markov chain Monte Carlo settings

Х $\square$ For hierarchical and complex designs, identification of the appropriate level for tests and full reporting of outcomes

$\bigotimes \square$ Estimates of effect sizes (e.g. Cohen's $d$, Pearson's $r$ ), indicating how they were calculated

Our web collection on statistics for biologists contains articles on many of the points above.

\section{Software and code}

Policy information about availability of computer code

Data collection No code was used to collect data.

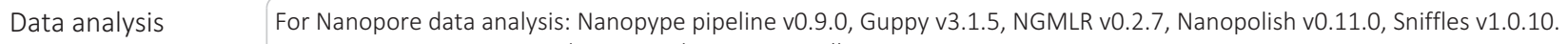

For WGBS processing: cutadapt, Picard, MOABS mcall, BSMAP.

For RNA-seq processing STAR, Stringtie v1.3.

For data analysis: Homer, BedTools, SamTools, R version 3.4.2, R packages edgeR for RNA analysis, HBS-tools for hairpin analysis, vioplot for generating violin plots, and scales and fields for plotting in $R$.

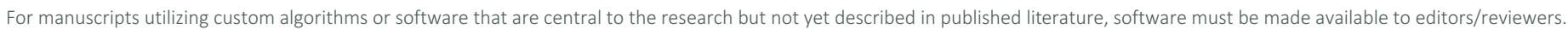
We strongly encourage code deposition in a community repository (e.g. GitHub). See the Nature Research guidelines for submitting code \& software for further information.

\section{Data}

Policy information about availability of data

All manuscripts must include a data availability statement. This statement should provide the following information, where applicable:

- Accession codes, unique identifiers, or web links for publicly available datasets

- A list of figures that have associated raw data

- A description of any restrictions on data availability

Data have been deposited in the Gene Expression Omnibus (GEO) under accession GSE126958. Other published datasets used in this study include: HUES64 DNMT3A single knockout ESCs (GSM1545005), and DNMT3B single knockout ESCs (GSM1545006) and mouse WT ESCs (GSM2339908). 
Please select the one below that is the best fit for your research. If you are not sure, read the appropriate sections before making your selection.
$\bigotimes$ Life sciences
Behavioural \& social sciences
Ecological, evolutionary \& environmental sciences

For a reference copy of the document with all sections, see nature.com/documents/nr-reporting-summary-flat.pdf

\section{Life sciences study design}

All studies must disclose on these points even when the disclosure is negative.

Sample size No sample size calculation was performed. We generally sequenced only one sample from each genetic background under the rationale that we required high sequencing depth to perform per-CpG analysis (such as DMR-calling). For our purposes, this strategy was preferred rather than generating several low coverage WGBS datasets that would be less informative or useful at the same cost.

For important samples that we performed detailed analysis on, we chose to sequence more than one sample. For DKO cells we sequenced three biological replicates (different clones) and for one clone two different passage numbers. For PKO cells we sequenced two difference passage numbers.

Data exclusions No data was excluded.

Replication We generated three biological replicates for the human DKO ESCs. There were no other attempts at replication.

Randomization Randomization was not relevant to this study as there were not different treatment options or conditions. We studied the impact of precise genetic mutations on global DNA methylation changes.

Blinding Blinding was not needed in this study as outcome (genomic changes in DNA methylation) could not be affected by the analyst knowing sample types.

\section{Reporting for specific materials, systems and methods}

We require information from authors about some types of materials, experimental systems and methods used in many studies. Here, indicate whether each material, system or method listed is relevant to your study. If you are not sure if a list item applies to your research, read the appropriate section before selecting a response.

\begin{tabular}{|c|c|c|c|}
\hline \multicolumn{2}{|r|}{ Materials \& experimental systems } & \multicolumn{2}{|c|}{ Methods } \\
\hline $\mathrm{n} / \mathrm{a}$ & Involved in the study & $\mathrm{n} / \mathrm{a}$ & Involved in the study \\
\hline$\square$ & $\bigotimes$ Antibodies & Х & $\square$ ChIP-seq \\
\hline 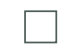 & $\bigotimes$ Eukaryotic cell lines & Х & $\square$ Flow cytometry \\
\hline$\bigotimes$ & $\square$ Palaeontology & Х & $\square$ MRI-based neuroimaging \\
\hline$\square$ & $\bigotimes$ Animals and other organisms & & \\
\hline$\bigotimes$ & $\square$ Human research participants & & \\
\hline$\bigotimes$ & $\square$ Clinical data & & \\
\hline
\end{tabular}

\section{Antibodies}

Antibodies used

supplier name, catalog number, clone name, lot number and dilution.

Human DNMT3A: Abcam, cat\# ab188470, Anti-Dnmt3a antibody [EPR18455], lot:GR224165-13, 1:1500 dilution

Human and mouse GAPDH: Cell Signaling, cat\# 2118, GAPDH (14C10), lot:10, 1:2000 dilution

Mouse DNMT3A: Abcam cat\# ab188470, Anti-Dnmt3a antibody [EPR18455], lot:GR224165-2, 1:2000 dilution

Mouse DNMT3B: Abcam, cat\# ab176166, Anti-Dnmt3b antibody [52A1018], lot:GR3199224-3, 1:500 dilution

Mouse DNMT1: Abcam, cat\# ab87654, Anti-Dnmt1 antibody, lot:GR3194562-7, 1:1000 dilution

Mouse Histone H3: Abcam, cat\# ab1791, Anti-Histone H3 antibody - Nuclear Loading Control and ChIP Grade, lot:GR293197-1,

1:10000 dilution

Validation

We compared expression levels between wildtype cells and those with genetic mutations (and therefore no expression) of the gene which shows specificity. You can see in our manuscript Extended Data Figures 1c, 6b and 8b all show both a positive and negative result that matches our genetic manipulations and shows specificity of the antibody.

\section{Eukaryotic cell lines}

Policy information about cell lines

Cell line source(s)

Human HUES64, HUES8. Mouse ESC, EpiSC 


\section{Mycoplasma contamination}

Commonly misidentified lines (See ICLAC register)
All cell lines tested negative for mycoplasma

No commonly misidentified cell lines were used.

\section{Animals and other organisms}

Policy information about studies involving animals; ARRIVE guidelines recommended for reporting animal research

Laboratory animals
Wild animals
Field-collected samples $\quad$ This study did not involve wild animals.
Ethics oversight $\quad$ This study did not involve samples collected from the field.
Harvard Universities ESCRO approved the ESC work and the IACUC (\#28-21) covers the mouse work.

Note that full information on the approval of the study protocol must also be provided in the manuscript. 